\title{
HR AND ETHICS. A RELATIONSHIP THAT IS WORK IN PROGRESS
}

\author{
BY
}

\section{SALONI PANDEY}

\begin{abstract}
A Thesis submitted to the Victoria University of Wellington
in partial fulfilment of the requirements for the degree of Master of Commerce
\end{abstract}

Victoria University of Wellington

2019 


\section{Table of Contents}

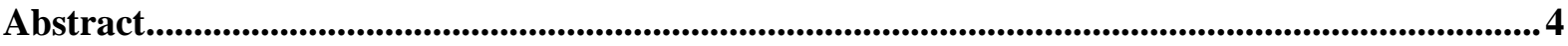

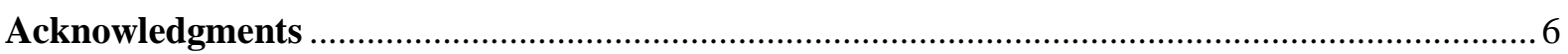

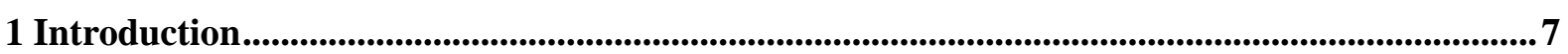

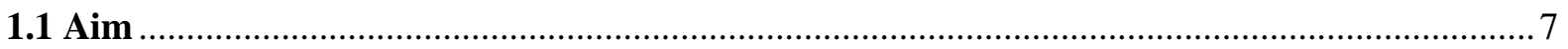

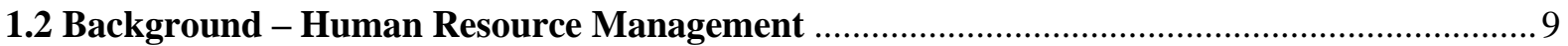

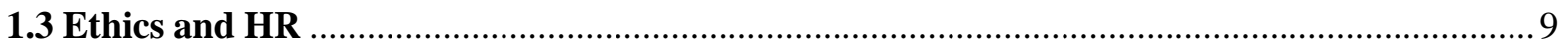

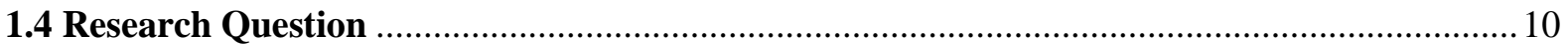

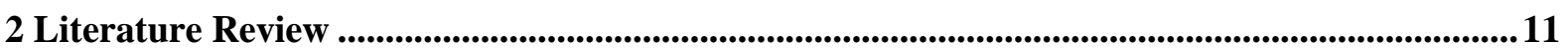

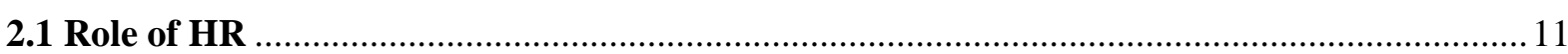

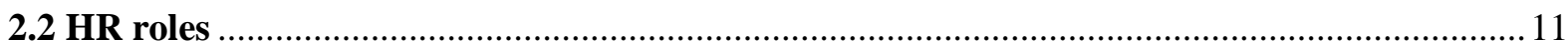

2.2.1 Administrative Expert ................................................................................................................ 11

2.2.2 Strategic Business Partner........................................................................................... 12

2.2.3 Change agent ....................................................................................................................... 12

2.2.4 Employee Champion ........................................................................................................... 13

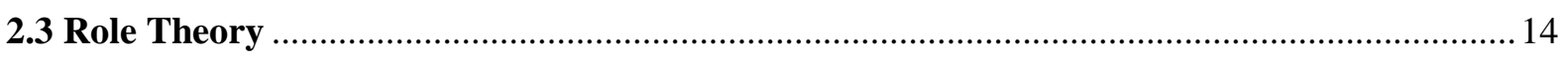

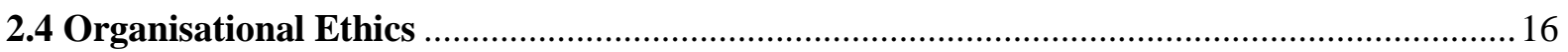

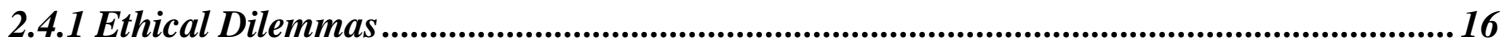

2.4.2 Moral Development Theory ....................................................................................................... 17

2.4.3 Ethical Culture and Leadership .......................................................................................... 18

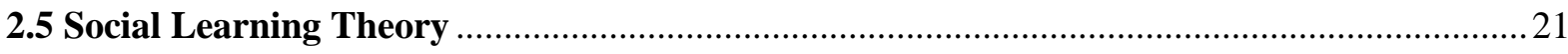

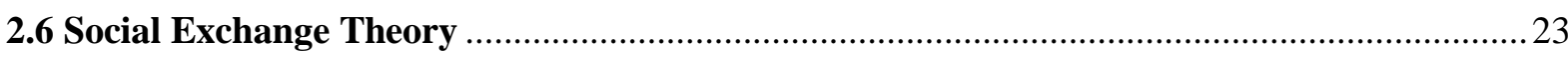

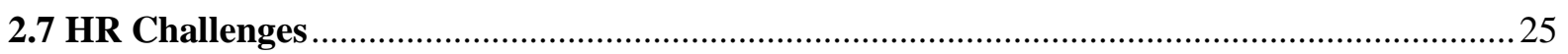

3 Methodology ............................................................................................................................................................28

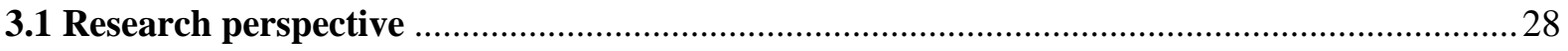

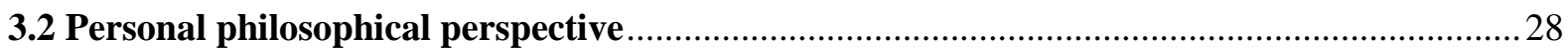

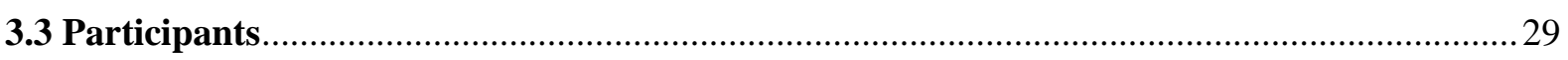

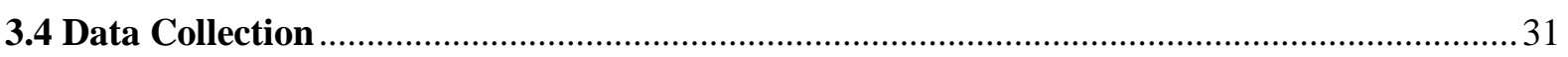

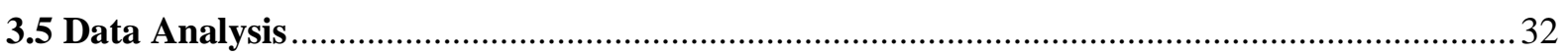

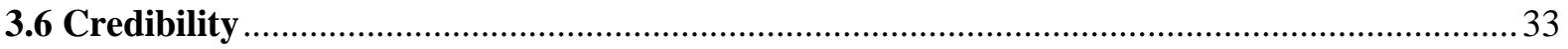

4 Findings and Analysis ................................................................................................................................34

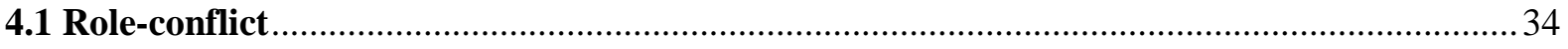

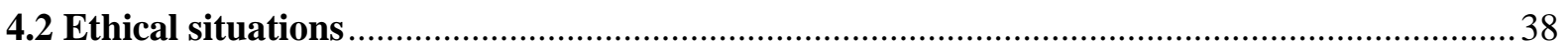

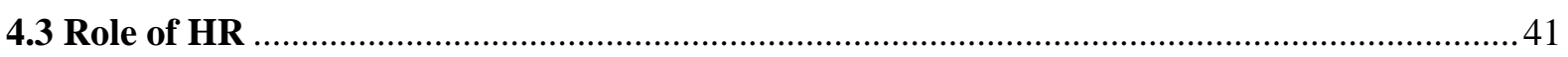

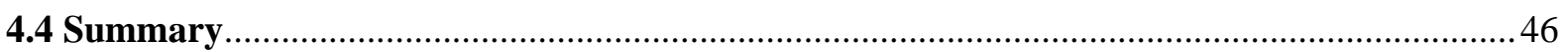

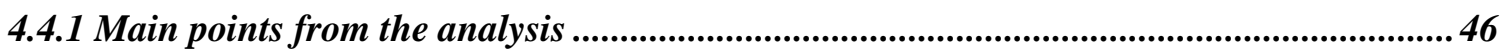

4.4.2 Summary of the Analysis ...........................................................................................................51 
5 Discussion \& Conclusion ..............................................................................................................................53

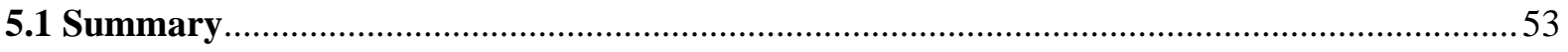

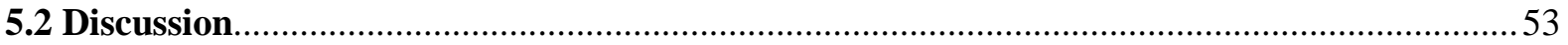

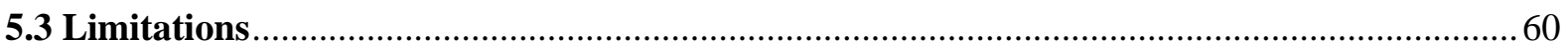

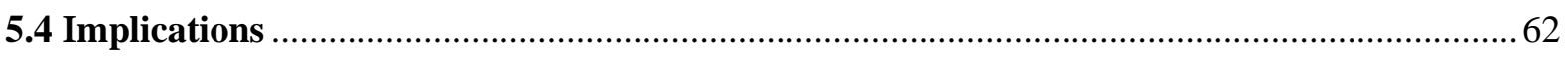

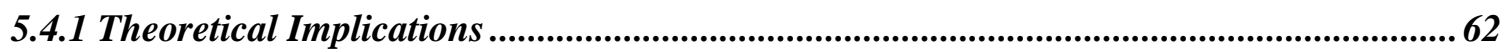

5.4.2 Practical Implications .............................................................................................................66 6

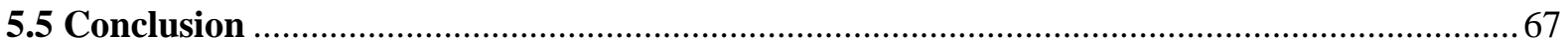

6 References..............................................................................................................................................69

7 Appendices.................................................................................................................................................... 76

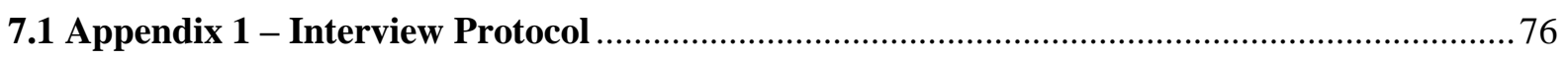

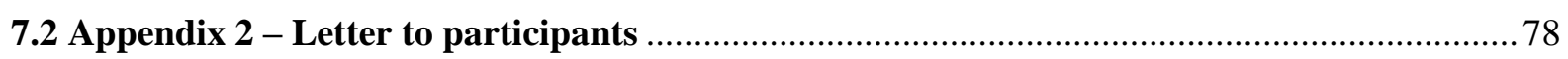

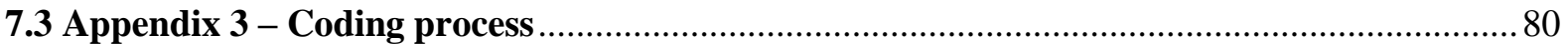

Figure $1 \mathrm{HR}$ function in an ethical organisation ......................................................................................63

Figure 2 HR function in an unethical organisation.............................................................63

Figure $3 \mathrm{HR}$ function in the ideal organisation .........................................................................................65 


\section{Abstract}

In this ever-changing business world, the role of HR has become significantly imperative due to the increasing focus on aligning people of the organisation with the overall business strategy, particularly in an era when unethical behaviour is not tolerated. However, considering the complexity of the HR profession, it has been questioned what the role of HR is. With the changing future of work, this question has become more prevalent considering the influence of factors such as globalisation, automation and generational changes. Various scholars have claimed that HR professionals should be undertaking four distinct roles of administrative expert, strategic business partner, change agent and employee champion, which consequently leads to a role-conflict for HR professionals, hence influencing their decision-making within organisations, particularly in ethical situations. Using Ulrich (1997) model as a benchmark, this thesis aims to explore the relationship between HR and ethics, focusing on the role-conflict that HR professionals experience in organisations, along with the best practices they use to cope with the role-conflict in ethical situations and the influence of these best practices on the future of HR.

Employing a qualitative method approach, this study uses in-depth semi-structured interviews with top-tier HR professionals working in organisations who are continually striving to build their ethical stance. The sample of this study was particularly important, as it was crucial to choose HR professionals who would make strong subject matter experts and provide rich and in-depth perspectives with regards to working in HR. The findings indicate that though there is a visibility and recognition of role-conflict within the profession, it wasn't regarded as a strong issue compared to what was reflected in the literature. Furthermore, support from the organisation leaders and a strong organisation culture along with following a fair, and consistent process allows for the role-conflict to be diminished, particularly in an ethical situation. The thesis also found that the profession is increasingly becoming more strategic, with the operational HR duties delegated to the line managers, and hence illustrating the emergence of two new roles of 'mentor' and 'analyst'.

The study contributes to the existing literature by proposing a new model for the HR profession by considering the various roles they are required to undertake and the significance for all the roles to work concurrently with each other for HR to truly succeed. Several implications such as re-training and a creation of an independent body for HR professionals, along with a strong organisational culture that allows HR to thrive, and the 
recognition of them gaining a seat at the management table are discussed, followed by an overall conclusion of what the future of the HR profession is. 


\section{Acknowledgements}

To my parents, Ranjan and Karuna, thank you for the constant reminder that I have what it takes to get this thesis done.

My younger, yet the smarter sibling, Symraina. Thank you for inspiring me every day to never give up. I am so grateful to have shared this Masters journey with you. You are going to be one remarkable influence within the Public Health sector.

Alastair. You have been my absolute rock. Thank you for pushing me to remain positive, to look at the opportunities waiting ahead and for the love and support every single day. Your kindness and patience (especially with statistics) have been so invaluable. I am looking forward to our next adventure.

Jenny, Olivia, Ved \& Jaymesh. I am honestly unsure where I would be without you guys. Thank you for the continuous reminder that I must aim high and go after the things I really want, but to also appreciate the small things when things don't go as planned. To all my friends, thank you for the endless support. I owe you guys!

Oliver and Ellis. We got through our Masters together with a lot of caffeine, tears and meme tags. Thank you for understanding the unexplainable tantrums and for providing distractions when I really needed it.

Dr Geoff Plimmer, thank you for your help with the qualitative methodology and for reviewing the draft. Your expertise is so appreciated. Victoria Beckett, thank you for all your advice during this process, and for such a warm welcome to the Brian Picot Chair Ethical Leadership team.

Professor Karin Lasthuizen. The most important thank you. This thesis would not have been possible without your guidance and wisdom. Your passion for ethical leadership is so inspiring, and it's only made me more interested in learning about the subject. Thank you for giving me the extra push that I really needed, but for also reminding me to pace myself and take a break. Thank you for being an amazing supervisor and an even greater mentor. Lastly, to all the participants. There wouldn't have been a research without your time and interest in my thesis. Thank you for your expertise and willingness to help. 


\section{Introduction}

\subsection{Aim}

The business world is rapidly changing due to various environmental and societal influences which is increasingly making organisation ethics an important component for organisational success. "Ethics can be defined as a set of principles and values that provide a context for the right action and the individual acts in accordance with that set of principles" (Lawton, Rayner \& Lasthuizen, 2013, p. 2). Therefore, in an organisation context, this set of values will enable the employees to comprehend the behaviours that are acceptable and how issues will be handled if there was an incident that involved unacceptable behaviour. It is, however, important to recognize that achieving ethical leadership is not easy considering the extent of accountability required. "To be an ethical leader is indeed to be different, as the leader acknowledges the complexity of running a responsible business, yet tries to do it anyway" (Schindler, 2019). Traditionally, the human resources function in organisations has been responsible for employee-wellbeing and behind the scene administration work, however this view of HR is increasingly changing as over the last decade, organisations have been aiming to make the HR function "leaner and more strategic" (Francis \& Keegan, 2006, p. 231) which arguably takes the focus away from employee and organisational well-being. More recently, scholars argue that building HR into a strategic role will take away the human characteristics from the HRM processes, hence making it alike to any other organisational function (Marchington, 2015, p. 177). Considering the changing business climate, the increasing awareness of ethical leadership and the remodelling of the HR function, the question remains on how HR professionals deal with ethics and what they can contribute to creating a wellrounded, ethical organisational climate.

The gradual changing nature of work is a positive change; however, it does offer various challenges for HR (Kowalski \& Loretto, 2017). Organisations are facing increasing changes in the workforce which ultimately requires organisation leaders to re-evaluate the organisation values and culture, along with the roles of different organisation functions, including HR. Additionally, the pace of change has enhanced, requiring companies to be quick-thinkers and responsive (Deloitte, 2016, p. 4) to meet the new demands, which consequently might put additional pressure on various functions within the organisation. Rise in globalisation and the developing artificial intelligence will eventually require people to upskill to keep up with the future changes while "organisations will need to comprehend the skills and abilities possessed by their current employees, so they adapt them to meet their 
changing needs" (IT Brief, 2019). Another significant factor contributing to the change in the workforce is the rise of the largest generational cohort of Millennials. "According to Deloitte, Millennials will make up 75\% of the global workforce by 2025" (Chung, 2019), who expect more than just a profitable outcome, emphasising strongly on values (Deloitte, 2016), hence illustrating a different kind of work ethic and organisational loyalty. It is evident that these changes will have a significant impact on organisations and in the way they operate.

Organisation leaders along with HR will have to ensure on strategizing accurately to manage the motivations, loyalties and work ethic of the future workforce to create an ethical culturally driven workplace. The challenge for the HR function would be to translate these new emerging changes into an appealing, but ethical workforce practice that also comply with the legal requirements (Deloitte, 2016).

The recent events at New Zealand's top law firm illustrate the uncertain relationship between HR and unethical situations. The investigation report of the law firm written by an external reviewer stated that "many people like but do not trust HR and would not approach them with an issue" (Bazley, 2018, p. 21) and that the behaviour of the wrongdoer was "potentially unchecked, over-looked and arguably rewarded" (Cribb, 2018). Furthermore, amongst the many recommendations for the firm, the report stated that HR at the law firm strongly lacked the expertise to support the kind of cultural change required at the firm (Bazley, 2018), hence making it visible that HR struggled significantly to manage the issue in an appropriate manner. The events at this law firm also reflected the global MeToo movement, focusing on victims of sexual harassment and encouraging them to speak out (Bushnell, 2019). The movement has had a significant impact on New Zealand businesses, requiring them to address workplace misconduct and initiate a crucial transformation in workplace culture. There is a possibility that the HR function at this law firm may have been conflicted due to the wrongdoer's higher position in the organisation, hence questioning the role and capability of HR professionals when an ethical situation arises in an organisation. The objective of this thesis is to explore the role-conflicts that HR professionals possibly may have to deal with when they are confronted with an ethical situation in the organisation and the influence it ultimately has on the HR profession. 


\subsection{Background - Human Resource Management}

Human Resources (HR) play an essential role in organisations to "address organisational challenges and contribute through effectively managing and rationalizing the employment relationship" (Ulrich \& Dulebohn, 2015, p. 188). They are responsible for several activities such as recruitment $\&$ selection, performance management, job design and training $\&$ career development (Ulrich \& Dulebohn, 2015), highlighting the demanding nature of the HR profession. Traditionally, HR professionals have been recognized as an "administrative, compliance and service-oriented" function within an organisation (Beer, 1997), however scholars argue that along with undertaking traditional roles such as 'employee champion' and 'administrative experts', HR professionals are also expected to become more strategic as a profession (Sheehan, De Cieri, Greenwood \& Van Buren, 2014) given the changing nature of business. The strategic role of HR professionals focuses on the move toward "human capital contributions, strategic capabilities and an organisation's competitive performance" (L. Lengnick-Hall, A. Lengnick-Hall, Andrade, \& Drake, 2009, p.69), hence demanding more than just an administrative and routine approach from the HR function. Scholars further argue that for HR professionals to become successful strategic partners, the operational HR tasks should be separated from the HR service (Beer, 1997). Considering this change, it is apparent that the role of HR has evolved, causing ambiguity for people who rely on the expertise of HR and for the HR professionals themselves. With the demand of juggling various roles along with the rise of globalisation, technology advances and diversity that influence organisation performance (Stone \& Deadrick, 2015), the literature says that HR professionals are increasingly experiencing role-conflict (Caldwell, 2003) hence hindering their ability to make comprehensive contributions to the overall organisation objectives.

\subsection{Ethics and HR}

With the increasing pressure on organisations to act responsibly and set out a visible code of conduct regarding ethical credentials (Parkes \& Davis, 2013), there is also an increasing pressure on HR professionals to ensure that appropriate care and duty are taken to create and promote an ethical organisational culture. Literature, however claims that "HR orientation often is absent from corporate ethics programs" (Weaver \& Trevino, 2001, p. 113), which is evident given the events at the top NZ law firm, further highlighting the impact of roleconflict that HR professionals experience. Alternatively, some scholars argue that though HR professionals are the ones who are expected to look after employee-wellbeing and ethical situations in organisations, this is not easy to maintain (Parkes \& Davies, 2013). Therefore, if 
the organisation promotes a culture of misconduct, it is extremely difficult for HR professionals to deal with an ethical situation. From a social learning perspective, ethical leadership within an organisation is influenced by leaders through the act of modelling (Brown, Trevino \& Harrison, 2005, p. 119), emphasizing that if a certain behaviour is modelled by the leadership team; this is likely to influence the behaviour of the employees working for the organisation. Hence, employees including the HR professionals observing the behaviours of their leaders will recognize the actions that are rewarded and the actions that require discipline. Considering this, it is imperative to understand that the HR professionals and their actions are likely to be heavily influenced by the everyday context under which they function. This has been supported by Foote \& Robinson (1999) who claim that "the extent to which the HR professionals are able to influence the organisational ethics is highly contingent upon the culture and structure of the organisation" (p. 96).

\subsection{Research Question}

Considering the ambiguous relationship between HR and ethics, this project will focus on the following research question -

How do HR professionals cope with role conflicts when they experience ethical situations in organisations and how do these best practices contribute to the future of the HR profession?

The next section of the thesis will commence with a literature review in relation to the role of HR and ethical culture. It will also explain four main theories; role theory, social learning theory, social exchange theory and moral development theory to understand role-conflict, what influences an ethical culture and workplace behaviours and how people reason their decisions when resolving an issue. Chapter 3 will then explain the use of qualitative methodology along with the characteristics and size of the chosen sample. The findings will be explained using suitable themes derived out of the semi-structured interviews. To conclude, the thesis will provide a concise discussion of the findings and practical implications for organisations and HR professionals. 


\section{Literature Review}

\subsection{Role of HR}

Traditionally known as personnel management (PM) that viewed "employees as expenses" (Ulrich \& Dulebohn, 2015, p. 190), PM eventually evolved into HR with a view that employees are resources that should be developed in conjunction with other organisational resources (Ulrich \& Dulebohn, 2015, p. 190). However, due to the various demands of HR, HR professionals are often confronted with a conflict regarding their role and expectations. According to Caldwell (2003) "HRM as an only solution to integrate business strategy and people management has created a whole new range of role strains, professional challenges and managerial expectations that have underscored the gap between HR rhetoric and reality" (p. 984). Various scholars have categorised HR professionals into four distinct roles; administrative expert, change agent, strategic business partner and employee champion (Ulrich, 1997; Caldwell, 2003). Additionally, in an updated model, Ulrich \& Brockbank (2005) reviewed the roles to be as functional expert, employee advocate, strategic partner which includes the change agent role, and HR leader which is the amalgamation of all the roles, showing that "one is credible within the HR function and the ones outside of their function" (Sheehan et al, 2014, p. 118). For the purpose of this research, the original Ulrich (1997) model will be used to illustrate the different roles HR professionals must undertake as the model seems to be distinctive and clearly highlights on the four unique roles. Considering the numerous expectations of the HR function, it is increasingly becoming important for organisations to establish what employees and the management team expect from HR professionals and whether these expectations align with the expectations that HR professionals have themselves.

\subsection{HR roles}

\subsubsection{Administrative Expert}

This function of HR refers to ensuring that all the customary work in an organisation is completed in an efficient manner. Ulrich (1998) further adds that as administrative experts, HR is expected to improve the effectiveness of their own occupation and the organisation. Therefore, they are responsible for the smooth running of the organisation along with being solution-focused regarding the HR processes and the organisation goals (Ulrich, 1998). Additionally, Lemmergaard (2009) claims "the underlying notion of this role is that in considering employees as costs, a competitive advantage can be attained by reducing these 
costs and hence increasing efficiency" (p. 184). Considering the nature of the work of an administrative expert, competencies such as attention to detail, excellent communication skills and high level of technical knowledge is strongly essential (Lemmergaard, 2009). The attributes required to perform the role of administrative experts at a high standard proposes that not only do HR professionals have to efficiently improve processes, but the improvement needs to be continuous to accomplish high level of services and processes. Hence, the overall prominence of the administrative expert role is on 'how to' characteristics of providing a service (Caldwell, 2003) therefore enhancing their role in creating value by competently running processes which consequently affects employee's work ethic and organisation perception.

\subsubsection{Strategic Business Partner}

The aim of this function is to focus on the processes from a strategic perspective (Lemmergaard, 2009), and to structure the organisation in such a way that enables them to achieve the organisation goals in an effective and purposeful manner (Ulrich, 1998). It is therefore argued that the main objective for this HR function is to achieve a 'best fit' correlation between HR strategies and the overall organisational purpose rather than focusing on 'best practice' remedies (Lemmergaard, 2009). Considering that the focus is on adding value to the overall organisational goal, it is imperative for the HR professionals to work jointly with senior management and line managers in designing organisation strategies and implementing those strategies (Lo, Macky \& Pio, 2015, p, 2310). Given the nature of the strategic business partner role, it is evident that HR professionals at this level will be required to portray strong leadership skills and effective interpersonal skills to work productively with a diverse range of stakeholders. Lemmergaard (2009) states organisational awareness, ability to problem-solve, strong communication skills and high levels of stress tolerance as key competencies for a strategic human resource partner. These competencies further show the necessity for HR professionals to build and maintain a trusting relationship with employees, line managers, and organisation leaders.

\subsubsection{Change agent}

With the increasing influence of globalisation, technology advancement and diversity, organisations need to ensure that these changes are reflected in the organisation to successfully keep up with the changing demands. The primary difference between people who are successful and people who are unsuccessful in business is their capability to respond to organisational change (Ulrich, 1998). Additionally, the way people respond to change is 
also dependent on the way change is carried out in the organisation, hence highlighting change agent as another function for HR professionals. "Utilizing a strategic focus on people, the change agent function aims at managing transformation and change faced by organisations" (Lemmergaard, 2009, p. 185). The focus for the change agent is to ensure that the organisation has the right resources and capacity for employees to embrace and execute change (Lemmargaard, 2009). Ulrich (1998) argues that as change agents, HR professionals do not perform change themselves, but are required to ensure that the change is carried out in an effective manner for employees to successfully adapt to the changes. He further adds that HR professionals will be able to achieve this by using a change model which consists of asking "who, what, why and how" (Ulrich, 1998, p. 132) to assess the strengths and weaknesses to "guide, facilitate and deliver change successfully" (Caldwell, 2003, p. 1000). Considering that as change agents, HR professionals need to act as promoter of the change, attributes such as being able to influence and negotiate, acting in an honest manner, thinking outside the box and effective communication (Lemmargaard, 2009) are strongly essential for preparing employees for the change.

\subsubsection{Employee Champion}

Organisational success not only comes from the profit gained, but also from the employees who work to build the credibility of the organisation. With the continuous changes in the business setting, employees are working longer hours which consequently affects employee engagement and productivity. Considering the importance of employee engagement in organisations, Ulrich (1998) claims that HR professionals must become employee champions to ensure that employees are engaged and productive in their roles. The role of the employee champion is simply to look after the employees' well-being (Lemmargaard, 2009) and "to manage the psychological contract between the organisation and the employee" (Caldwell, 2003, p. 997). HR professionals as employee champions should ensure that line managers and immediate supervisors are trained to enhance the morale of their employees (Ulrich, 1998) which consequently will lead to high employee engagement. In addition to providing employees with the right set of training, scholars emphasise the role of employee advocate where HR professionals "must represent the employees to management and be their voice in management discussion" (Ulrich, 1998, p. 130). By being an employee advocate, employees are more likely to have confidence in the fact that HR professionals will voice their concerns, opinions and input to the management team. Given the nature of the employee champion role, HR professionals in this role will need to manage the relationship with the management team 
and represent the employees, hence qualities such as "interpersonal skills, strong communication, ability to teach and learn" (Lemmargaard, 2009, p. 186) are highly desirable to gain employee trust which accordingly will impact employee engagement.

\subsection{Role Theory}

The HR literature has highlighted the increasing emergence of HR professionals juggling different roles within organisations such as 'employee champion', 'strategic partner', 'change agent' and 'administrative experts', hence creating ambiguity regarding their role and expectations. Role theory is concerned with the idea that "human beings behave in ways that are different and predictable depending on their respective social identities and the situation" (Biddle, 1986, p. 68). Biddle (1986) further emphasises that from an organizational role theory perspective, roles are associated with established social positions with specific norms and standards. Therefore, norms among individuals are likely to vary due to various demands and pressures from different groups in the organisation. Essentially, these differing norms lead to individuals having to deal with role-conflict. For this thesis, the definition of roleconflict by Rizzo, House and Lirtzman (1970) will be followed who state that role conflict is a result of inconsistent expectations and organisational demands causing stress and dissatisfaction within the organisation. Moreover, role conflict is also associated with inadequate job performances, lack of loyalty and commitment to the organisation and high rate of absenteeism (Biddle, 1986).

Role theory can be related to HR due to the increasing interest with regards to their function in an organisation. According to Shivers-Blackwell (2004), organisational culture and structure act as a direction for managers on how they comprehend their role to be. Therefore, for HR professionals who often hold a managerial position; the culture and context of the organisation is likely to add pressure on them to adjust their behaviour and actions in accordance with the organisation requirements. Due to the changing business world, each organisation is likely to have its own set of requirements, hence accompanying the changing behaviours of managers and employees. Furthermore, considering the different requirements of organisations, the role of HR varies from organisation to organisation. Scholars such as Ulrich (1997), Storey (1992) and Caldwell (2003) have discussed the various roles that HR professionals are expected to portray. The common theme that derived out of their models was the distinction between the 'operational' and 'strategic' roles that HR professionals often must comply with (Welch \& Welch, 2012). Storey (1992) distinguished HR role typologies into an advisor, changemaker, regulator and handmaiden (Welch \& Welch, 2012). Similarly, 
Ulrich (1997) proposed four roles for HR professionals as administrative experts, strategic business partner, change agent and employee champion (Foote \& Robinson, 1999). Lastly, though very similar to the models of Ulrich and Storey, Caldwell (2003) argues that his model of advisor, service provider, regulator and change agent (p. 992) is more reflective of HR's role considering the changes in the current business trends influenced by organisational restructurings (Welch \& Welch, 2012). The research also emphasised the rise of role conflict for HR professionals due to the demand to perform more than one role, which was underemphasized by Ulrich and Storey's model (Caldwell, 2003).

Shivers (2004) proposed that managers tend to have various leadership styles even when working in organisations with similar job contexts (p. 41). Hence, using role theory, the research by Shivers (2004) determined how managers' view of the organisation culture and structure affected their leadership style. Similarly, when applying this to HR managers in organisations; the culture of the organisation can determine how HR professionals perceive their role requirements to be within the organisation, which could be different to the way the senior management team perceive their role to be within the same organisation. Therefore, influencing both parties' leadership behaviours within the same context. Along with the context of the organisation, another factor to consider is the personality of the individuals who are in leadership positions. Role theory further claims that an individual's personal traits play a significant role in the way they interpret their role requirements and leadership qualities, which is further likely to influence their leadership behaviour (Shivers, 2004). Considering this, HR professionals in such instances are likely to experience increased roleconflict during an ethical situation as their personality and emotions might influence their decision-making, which may be different to the way the organisation expects them to resolve an ethical issue.

In recent times, role theory has been recognised to understand the different performance initiatives brought into organisations. Bush (2018) introduces the concept of triple bottom line focusing on the amalgamation of social, economic and environmental factors into the organisation's overall vision and goal (p. 1) and the effect it has on the employee's roles. This is particularly relevant to HR professionals due to the decision-making they must undertake when dealing with situations that might focus on one goal more than the other. At an individual level, this alignment is not always achievable due to differing priorities and values (Bush, 2018) hence further highlighting the issue of role conflict. Moreover, Bush (2018) says these role ambiguities are also shaped through organisational factors such as the support 
provided to individuals. Perceived organisational support (POS) has been regarded as one of the most fundamental factors that contribute to the employee role involvements as it enables employees to comprehend whether their work is valued by the organisation (Bush, 2018). Similarly, according to Chiu, Yeh \& Huang (2015) "social support in an organisation can be regarded as a 'social resource' which can deplete the effects of role-conflict and role ambiguity" (p. 311). Therefore, organisations that believe in high quality social and perceived organisational support will provide employees with strong confidence that the senior organisation members will deliver guidance during a complex situation (Bush, 2018). For HR professionals who are confronted with an ethical dilemma, this will be particularly useful due to the perceived power imbalance between themselves and the leadership team. Hence, the more support HR professionals are provided with, the more successfully they will be able to resolve an ethical situation.

\subsection{Organisational Ethics}

\subsubsection{Ethical Dilemmas}

One of the main struggles for organisations in recent times is distinguishing what constitutes as an ethical issue, which may ultimately lead to a dilemma for people who are responsible for resolving the ethical issue. According to Lawton et al (2013), "what is constituted as unethical behaviour varies from culture to culture and from country to country" (p. 3) due to the different legislations and societal expectations (Brian Picot Chair in Ethical Leadership, 2018). Therefore, along with focusing on the act itself, another imperative factor for organisations to engage in is how the act is conducted (Lawton et al, 2013) as that is likely to define the quality of the relationship between the perpetrator and the victim, the duty of care required from the employer and the acceptable norms within the organisation. Acts such as fraud and corruptions can clearly be described, however, acts such as sexual harassment and bullying are less obvious (Lawton et al, 2013, p. 3) hence causing ambiguity for organisations involved. With increasing pressure on organisational cultures, the nature of work and the role of a manager is dramatically changing as "organisations are now more assertive, competitive and insecure" (Rayner \& Harrington, 2010, p. 3), hence making unethical activities more tolerant. Consequently, when an ethical issue arises, the dilemma comes into play when professionals such as line managers or HR professionals are confronted with deciding between challenging moral duties or between competing beliefs about what the right thing to do is (Linehan \& O'Brien, 2017, p. 765); leading to them acting in less than preferred way to manage their personal and organisation expectations. Linehan \& O'Brien (2017) further add 
that for HR professionals, encouragement and power within an organisation is limited and with various demanding roles, they struggle to make accurate decisions when an ethical issue arises. Along with the different roles that is expected of the HR professionals, it is equally imperative to consider the everyday context under which they function. Organisational members are likely to be influenced by several factors such as "individual characteristics, organisational culture, workplace pressure and quality of relationships" (Linehan \& O'Brien, 2017, p. 765), further creating challenges for HR as they are likely to adjust their expertise, knowledge and values to align it with the organisational factors. With HR professionals responsible for the consequences of policies and practices involving organisational activities such as recruitment \& selection, performance management, and training and development, they are ultimately expected to manage complex human emotions while dealing with their own competing values and emotions. The imbalance between powerful emotions, organisational values and individual values represent the intricacies of dealing with ethical dilemmas as these factors trigger the intensity of the arising ethical situation.

\subsubsection{Moral Development Theory}

Ethical culture is strongly based upon the ethical leadership that is promoted within an organisation. To be considered an ethical leader, one should be caring, honest and reliable (Brown, Trevion \& Harrison, 2005). However, an equally important attribute that makes a leader ethical is the way individuals' reason through complex issues and resolve ethical dilemmas. According to Kohlberg (1969), how an individual makes their decision regarding an ethical issue is just as important as the behaviour that is authorized by an organisation or the general society. The moral development theory describes "the structures of reasoning that individuals apply when thinking through ethical issues and resolving ethical dilemmas" (Jordan, Brown, Trevino \& Finkelstein, 2013, p. 663). Hence, the focus is simply on moral reasoning rather than on actions because how an individual thinks about a situation may not necessarily result in same actions in an ethical situation (Jordan et al, 2013). There are three stages in moral development which differ from individual to individual and is based on social perception (Fraedrich, Thorne \& Ferrell, 1994). The pre-conventional stage is focused on the individual (Fraedrick et al, 1994, p, 834) and the moral reasoning is predominantly fixated on personal consequences with regards to an action ( $\mathrm{Li}$, Chao, Chen \& Zhang, 2018), therefore highlighting the significance of rewards and penalties. In contrast, conventional stage focuses on sustaining interpersonal relationships (Jordan et al, 2013) emphasising a collective nature of loyalty whereas the post-conventional level is exceedingly macro (Fraedrick et al, 1994). 
According to scholars, moral reasoning at post-conventional stage may promote ethical leadership more powerfully compared to the other two levels as it considers "justice, individual rights and wellbeing" (Jordan et al, 2013, p. 665), highlighting "a concern for the social system in general" (Fraedrick et al, 1994, p. 834) regardless of power imbalance. Considering the three stages of moral development theory, the high level of moral reasoning is strongly beneficial for HR professionals as it promotes fair and justice driven ethical leadership. Consequently, the prominence of high moral reasoning through conveyance or actions (Jordan et al, 2013) will influence HR professionals' moral reasoning as the ethical expectations will be visibly clarified. Though moral development theory simply focuses on moral reasoning rather than behaviour; research does suggest that "ethical reasoning is moderately associated with genuine ethical behaviour" (Jordan et al, 2013, p. 665) as various additional factors may influence this relationship. Using Kohlberg's moral development model, Trevino (1986) introduces an interactionist model focusing on the collaboration between individual and situational factors when making an ethical decision in an organisation. The author aims to illustrate several additional factors and the interaction between those factors which may influence an individual's moral reasoning and the actual behaviour in relation to an ethical dilemma in organisations (Trevino, 1986). "Individual factors such as ego strength, field dependence and locus of control associated with situational factors such as job context, organisational culture and characteristics of the work" (Trevino, 1986, p. 603) influences the way an individual reasons about an ethical situation and their ultimate action in resolving the ethical issue. Therefore, in conjunction with Kohlberg's model that highlights the reasoning element in an ethical situation, Trevino (1986) shows the significance of considering additional variables that consequently play a role in defining the reasoning and the actions taken to resolve the ethical issue. This is particularly relevant to HR as due to the role-conflict within the HR profession, it will increasingly become imperative for the HR professionals to deliberate all influencing factors to ensure an accurate decision is achieved in an ethical situation.

\subsubsection{Ethical Culture and Leadership}

Organisational culture can act as a key influential tool for employees to comprehend the accepted actions and behaviours. Employees look beyond themselves to other people with significant status to understand the norms and values of an organisation. "Therefore, in the workplace, leaders should be the central source of guidance" (Brown, et al, 2005, p. 117) illustrating that ethical culture is rooted from the leadership that is present in the organisation 
influencing other organisation functions such as HR. According to scholars, ethical culture can be defined as features of organisational behaviour that encourage organisations to function in a way that constitutes ethical or unethical behaviour (Riivari \& Lamsa, 2014) whereas ethical leadership is the illustration of adequate and expected behaviour through actions and interactive relations, and encouragement of these actions through strong communication, reinforcement and decision-making (Trevino \& Brown, 2006, p. 595). The correlation between ethical leadership and ethical organisation culture is well reflected in Kaptein (2011) Corporate Ethics Virtues (CEV) model which focuses on dimensions that are likely to enhance an organisation's ethical culture including components of ethical leadership. The model illustrates clarity, ethical role-modelling of management, the capability to behave ethically, commitment to behave ethically, visibility of ethical behaviour, openness to discuss ethical issues and reinforcement of ethical behaviour as essential dimensions of creating a strong ethical culture within an organisation. Therefore, ethical culture ultimately illustrates what the organisation believes in and the expected behaviour from the employees, which is often an influence of the modelled behaviour by the organisation's leadership team.

Scholars argue that "leadership team play a key role in providing an ethical context to the organisation employees and in shaping the collective character of the organisation" (Neubert, Carlson, Kacmar, Roberts \& Chonko, 2009, p. 157) as employees are likely to look to their superiors to recognize the encouraged behaviour along with the behaviour that will be punished. Applying the social learning theory, Brown et al (2005) say for leaders to be deemed ethical, they should be perceived as "attractive, credible and legitimate" (p. 120). Additionally, leaders who strive to promote and create an ethical climate within the organisation will gain the employees' attention regarding the importance of organisation ethics. In conjunction with this, Neubert et al (2009) argue that the impact of ethical leadership also extends toward employee work ethic and their attitudes towards organisation and its values which is particularly relevant for HR professionals as it might provide them with clarification of what is expected from them. It is then likely to have an impact on the organisational culture which can further be enhanced or diminished by the organisation's ethical leadership.

Considering the influence of ethical leadership on employees and the organisation culture, organisation leaders will first need to establish themselves as an ethical leader for employees to be influenced. Trevino, Hartman \& Brown (2000) explain the "dual approach to ethical leadership with an emphasis on reputation for ethical leadership' (p. 29). This emphasis on 
reputation illustrates the importance of perception by the employees on ethical leadership within the organisation, as that would ultimately influence the employee's work and attitude. By interviewing senior executives, the authors explain how being perceived as a moral person and a moral manager leads to a reputation for ethical leadership. The study found that to be regarded as a moral person, one should have the right attributes, embark in specific kind of behaviours and make decisions based on ethical standards, and to be regarded as a moral manager, one should role-model the ethical behaviours, consistently communicate the importance of ethics and reward employees for complying with the required ethical standards (Trevino et al, 2000). Consequently, the combination of two delivers a certain kind of reputation for ethical leadership, hence "a strong moral person and a strong moral manager produces an ethical leader" (Trevino, et al, 2000, p. 137). The study essentially demonstrates the significance of having a reputation of being an ethical leader as to be an ethical leader, one needs to be perceived ethical by people who will be influenced by this leadership.

It is evident that ethical leadership is highly crucial for organisations, particularly in an era when the business climate is rapidly changing. Therefore, "values are the glue that can hold things together, and values must be conveyed from the top of the organisation" (Trevino \& Brown, 2006, p. 128). Considering the emphasis on the increasing need for ethical leadership and the changing business climate, it is imperative to understand how different organisations perceive ethical leadership, therefore influencing their ethical leadership style. As explained by Heres \& Lasthuizen (2012), ethical leadership differs from organisation to organisation, hence showing that one type of ethical leadership style doesn't fit all organisations. Data derived from semi-structured interviews with managers working in various organisations, the researchers found that interpretations on ethical leadership differ slightly between public, private and hybrid organisations (Heres \& Lasthuizen, 2012). For example, the study showed that "managers of public and hybrid organisations emphasised that ethical leadership requires responsiveness to societal demands whereas private sector managers described ethical leadership is something that concerns internal organisation and its members" (Heres \& Lasthuizen, 2012, p. 452), illustrating the difference in interpretation between public and private organisations. Therefore, considering the findings from Heres \& Lasthuizen's (2012) study, it is evident that though the basic factors of ethical leadership are consistent, the way these factors are construed and represented may differ from context to context. As the business climate changes, it is likely that components of leadership and ethics will differ as 
organisations compete to meet the new demands while striving to find and retain the high performing employees.

\subsection{Social Learning Theory}

Social learning theory has gained recognition within the ethics literature to explain the role of ethical leadership and its impact on the organisational culture. Within the ethics and organisation context, Brown, et al (2005) proposed that from a social learning perspective, "leaders influence the ethical conduct of followers via modelling" (p. 119). Therefore, employees observing the behaviours of the organisation leaders will enable them to recognize the behaviour that will be rewarded and the behaviour that will require discipline. Additionally, modelling may potentially help employees learn the code of conduct and the culture of the organisation via observing actions rather than comprehending a written document which may not transfer into actions (Brown et al, 2005). Considering this, Brown et al (2005) further argue that leaders are an essential source of providing an ethical organisational culture through modelling due to their high-status roles, success in the organisation and the power to encourage behaviour of others (p. 119). The theory can be related to HR professionals considering they are often responsible for setting the code of conduct for the organisation which is heavily influenced by the top management team. Hence, they are likely to observe the actions of the senior managers, particularly during an ethical situation to understand what is acceptable and what is regarded as misconduct.

However, an issue arises when the behaviour of organisation leaders is unethical in the view of HR professionals, therefore adding pressure on HR professionals to act in accordance with the behaviour set by the management team. In instances such as this, it can be argued that along with ethical leadership, social learning theory can also influence unethical behaviour due to the modelled behaviour of the organisational leaders (Brown et al, 2005). Establishing bullying as an ethical issue, Harrington \& Rayner (2010) say that "HR professionals' actions and decisions appear to be motivated more by organizational and personal factors than by fairness and ethics" (p. 7), as the demands to meet organisational requirements may lead to actions that support management's decision-making behaviour.

The phenomenon of vicarious learning through modelling is increasingly discussed in the social learning theory literature. Learning, defined as "a relatively permanent change in behaviour" (Manz \& Sims, 1981, p. 105) is strongly associated with people's work behaviour which is formed through observing the behaviours of their superiors in a work setting. Hence, the nature of modelling is a form of vicarious learning that plays a leading role in social 
learning theory (Manz \& Sims, 1981). Manz and Sims (1981) further argue that when employees observe a notable behaviour, the action from the observer at times may not be executed if there is a lack of motivation. Therefore, implying that motivation plays an important role in the observer's decision to change their behaviour to align it with the actions they have observed. Consequently, for modelling to have a significant impact on employees' perception of acceptable actions, models themselves need to be the right kind of models. Hence, for successful vicarious learning to occur, leaders need to be influential and sincere about their actions on day to day basis, rather than sporadically (Brown et al 2005, Manz \& Sims, 1981).

In the more recent social learning theory literature, the concept of vicarious learning has been further investigated to emphasise the informal forms of employee training. Some scholars have argued that modelling and behavioural observation tend to be more effective for employees to learn the norms that shape the organisational culture rather than the traditional forms of training such as workshops and seminars (Cialdini, 2003). A recent study by Sumpter, Gibson \& Porath (2017) explains how vicarious learning can be utilised to train employees to behave autonomously and expediently. Their research confirmed that "autonomous and expedient behaviour of a supervisor both increases the extent to which employees behave similarly and is associated with enhanced employee performance" (Sumpter et al, 2016, p. 140). Hence it is evident that through modelling, not only do employees learn the accepted behaviour which will influence future decisions, but also enhance employee performance which ultimately leads to a workforce that experiences continued high performance.

In conjunction with learning the norms through observing the behaviours of others, employee voice regarding improvements and dysfunctionality within an organisation is a significant component of social learning theory. According to scholars, employee voice is defined as a form of organisation behaviour where employees communicate productive, change-oriented recommendations to make necessary improvements (Avey, Wernsing \& Palanski, 2012). The aim of many organisations is to ensure that the employees are comfortable to voice their opinions, however, this is often problematic to achieve. Based on views of social learning theory, the ethical climate can encourage followers' behaviours as the team climate sets a pattern of accepted behaviour that members of the team are expected to follow (Bai, Lin \& Liu, 2017, p. 7). Bai et al (2017) illustrate that a shared, team-oriented observation of ethical climate can influence the behaviour of the employees due to environmental factors and social 
cues implemented within the team. Furthermore, due to the climate set by the leaders, employees will either feel comfortable to voice their opinions or shy away from it.

For HR professionals, this collective ethical perception is highly imperative due to the emphasis on the 'collective' and 'team-level' nature. Considering Bai et al (2017) argument, if the ethical climate is evident within an organisation and employees consequently voice their thoughts, HR professionals may then have the power to deal with arising ethical situations with ease as every team member is likely to be aware of what constitutes as acceptable organisational behaviour. The individual and team-level views of social learning theory highlight the "synergy between individuals and the environment" (Bai et al, 2017, p. 23) implying that organisational employees are likely to regard managers as role models in an environment that strongly believes in ethical practices (Bai et al, 2017). This suggests that though ethical role modelling is highly needed to influence employees, the team climate created by organisation leaders is equally influential. Hence, a climate where managers utilise robust HR practices such as performance-based reward structures, training and development schemes, and clear articulation of punishment for misconduct actions, stresses the importance of expected ethical actions and behaviours from employees (Bai et al, 2017). Considering the characteristics of SLT, the theory is relevant to this research as it might provide an insight into the influence of the organisation leadership on the HR function and the way they deal with role-conflict.

\subsection{Social Exchange Theory}

In everyday lives, relationships are usually built through social exchanges that evolve over time to either strengthen or weaken relationships. This is also applicable within an organisational setting, where the degree of social exchanges between an employee and manager indicates the quality of a relationship which ultimately affects the organisation culture and behaviour. According to scholars, social exchange theory (SET) is a significant theory for comprehending workplace relations and behaviour (Cropanzano \& Mitchell, 2005). Deriving from various fields such as anthropology, sociology and psychology, the common theme amongst all theorists seems to be that "SET involves a series of exchanges that produce obligations" (Cropanzano \& Mitchell, 2005, p. 874). Furthermore, these exchanges have the potential to create high-quality relationships which will likely influence employee performance and behaviour. This theory is particularly useful to understand workplace relationships when there is an ethical situation within an organisation. 
Using SET concepts of organisational justice, psychological contract, and perceived organisational support (POS), Parzefall and Salin (2010) explain the issue of workplace bullying. Organisational justice typically refers to the perception that employees have of fairness within the workplace with interactional justice being one of the dimensions which involve truthfulness and respect (Parzefall \& Salin, 2010). Therefore, when an employee witnesses a bullying incident even if they are not the direct target, it will influence their perception of the organisational exchange which will further influence the organisational climate. Additionally, when an employee is a victim of bullying from their superior, the employee is likely to view this as a breach of the psychological contract and "violation of the norm of reciprocity that governs the employee-employer exchange relationship" (Parzefall \& Salin, 2010, p. 769). Due to the reciprocity of this contract, one employee's actions are often dependent on the other employee's or manager's behaviours (Cropanzano \& Mitchell, 2005). Lastly, when an employee perceives the organisation to be supportive and dedicated to appropriately deal with misconduct, the employees will reciprocate this by performing at the highest standard to achieve the organisation's goals (Parzefall \& Salin, 2010). This is further supported by Cropanzano and Mitchell (2005) who say that POS has been extensively explored as a consistent strong indicator of commitment (p. 884) which in due course affects the employee's work ethic with regards to organisational outcomes. Reciprocity is an imperative component in social exchange theory as it emphasises "unspecified obligations" (Cropanzano \& Mitchell, 2005, p. 882) which affect future employee and employer behaviour which ultimately influences workplace culture.

Considering the importance of reciprocity in SET, organisation and employees need to work together to achieve a relationship that is reciprocal, evidently leading to a high-quality working relationship. Scholars argue that trust is viewed as an essential factor reinforcing quality relationships (Gould-Williams \& Davies, 2005, p. 4) as a trust-worthy relationship between an employee and the organisation will result into benefits for both the parties. Social exchange theory is particularly applicable with regards to trust as it helps to illustrate when one party is prepared to trust another party rather than inflict control over the other party (Whitener, Brodt, Korsgaard \& Werner, 1998). Furthermore, SET literature suggests that leaders of the organisation play a fundamental role in exchange process as employees learn the norms and organisational values from their behaviours, hence suggesting that if employees perceive the actions to be positive, they will likely respond with attitudes that align strongly with the organisational goals (Gould-Williams \& Davies, 2005). Moreover, 
this characteristic of SET aligns with social learning theory as it proposes that within an organisational context, employees learn about organisation culture and values by observing the behaviours of the leaders.

The concept of trust can closely be associated with the Leader-Member exchange (LMX) theory which focuses on a reciprocating relationship between a leader and the follower. The theory is strongly based on the social exchange theory, as it suggests that a "relationship progresses between the supervisors and their employees against the background of a formal organization" (Wayne, Shore \& Liden, 1997, p. 83). In these exchange relationships, parties involved engage in behaviours that go above the scope of their day to day job expectations (Wayne et al, 1997), hence encouraging both parties to treat each other in a fair manner where duties are reciprocated and will benefit both the parties in future. In recent literature, Choi, Kim \& Yoo (2016) say that when the LMX is of a higher quality, employees are more likely to work harder, display a greater commitment to the organisation and effectively perform their roles, ultimately influencing the organisation culture. The emphasis on relationships is one of the main features of the SET, therefore making it appropriate for HR professionals as it shows the quality of relationship they have with various organisation members which consequently sheds light on what is expected from them, and the process they use to resolve ethical situations.

\subsection{HR Challenges}

Given differing roles that are expected from HR professionals, and the increasing need for ethical organisation culture, Linehan \& O'Brien (2014) argue that the complexity of the HR profession and the competing demands leads to emotional labour which can be regarded as one of the key challenges for HR professionals. They further argue that due to the various expectations, the influence and power of HR professionals in organisations are limited as they are restricted by circumstantial pressures (Linehan \& O'Brien, 2014). This shows the visibility of role-conflict within the profession along with the uncertainty of the role as it appears that the HR function is highly contingent upon how the organisation utilises HR (Foote et al, 1999). The findings from their study demonstrate that managing emotions is part of HR work, showing that HR professionals should "act as emotional managers, who control and contain emotions of themselves and others" (Linehan \& O'Brien, 2014, p. 1277). Furthermore, the authors claim that HR professionals are expected to portray a range of emotional fronts toward different organisation members to maintain credibility (Linehan \& O'Brien, 2014). The study aligns with the claims made by scholars such as Caldwell (2003) 
who say that there is a degree of role tension within the HR profession due to the duality and ambiguity in the profession, however it also adds a new perspective of emotional labour and emotion management being fundamental to handling the complexities that occur due to the ambiguity in the profession (Linehan \& O’Brien, 2014).

From a different perspective, in relation to the various roles that HR professionals must undertake, additional scholars have researched whether one role is more beneficial for organisation performance than the other. Brown, Kulik, Cregan \& Metz (2017) focused their research on the role of HR with regards to employee change cynicism as a result of any kind of change within the organisation. Focusing on the administrative expert role, and the strategic change agent role, the aim of the research was to investigate which form of HR role was best suitable for executing a change within the organisation that will best decrease employee change cynicism. As HR is usually regarded as the information source in an organisation (Brown et al, 2017), it is imperative for organisations to ensure that HR professionals provide relevant information with regards to the rationale behind the change. As change agents, HR professionals ensure that the change is executed by addressing queries with regards to the change (Ulrich, 1998) and they work meticulously with line managers to ensure that they deliver positive messages about the change (Brown et al, 2017).

Alternatively, in an administrative expert position, HR focus on "process efficiency" (Brown et al, 2017, p. 8), hence they are not directly involved in change management, making it difficult for them to communicate the reasoning of the change. Considering the difference between the two roles and their impact on change within the organisation, Brown et al (2017) found that the strategic change agent role was more effective in decreasing employee change cynicism than administrative expert role. This was due to the lack of information provided to the employees with regards to the change when HR worked as an administrative expert. The study shows the emerging importance for HR professionals to become more strategic and gain more authority within the organisation in relation to change management and employee satisfaction.

Similarly, Kuiper \& Giurge (2017) conducted research to "illustrate the influence of the operational and strategic HR role to organisation performance by considering the organisational strategy" (p. 3192). After interviewing HR professionals, the researchers found that the operational HR role does not add any value to the organisation performance, however, they are valuable for the everyday efficient running of processes and delivering accurate practices and policies (Kuiper \& Giurge, 2017). This finding aligns with the research 
findings of Brown et al (2017) as they found that the administrative expert role, an operational role didn't contribute positively to change management. Furthermore, the study concluded that by "developing practices and behaviours to sense and seize opportunities for change and innovation, the strategic HR role can enhance organisation performance if a strong organisation strategy was lacking” (Kuiper \& Giurge, 2017, p. 3196). The findings from this study illustrate the increasing need for HR to gain an in-depth understanding of the organisation strategy, to contribute their knowledge and enhance firm performance. Aligned with the contribution by Brown et al (2017), this study further challenges the traditional role of $\mathrm{HR}$ and confirms the changing nature of HR profession, with a greater emphasis on the strategic HR role as the main driver for organisation and employee performance.

Considering the focus on strategic versus operational HR, there are several challenges facing the HR professionals given the complexity of their role. Legge (1978) argued that HR professionals face several uncertainties in their role such as their problematic position being part of the management team, but also having responsibility for the employees and the difficulty in determining their contribution to various situations, hence the ambiguity in explaining their success. Decades later, through in-depth interviews with senior executives, Guest \& King (2004) concluded that challenges with regards to "how far to act as a professional or a manager, whether to do the right thing according to what is known about best practice or to do what is best from a more sectional management perspective" (p. 419) added further uncertainties for HR professionals, illustrating the evolving, but demanding nature of the profession. The continuous ambiguity also raises questions of power (Guest \& King, 2004), due to the growing pressure for the HR profession to become more strategically aligned with the organisation's management team. Moreover, these challenges and the increasing range of complex situations (Guest \& Woodrow, 2012) also hinder HR professionals' ability to achieve and implement ethical goals within an organisation. Due to meeting the demands of various organisation members, "organisation ethical code of conduct can become bureaucratised due to the absence of moral impulse" (Guest \& Woodrow, 2012, p. 117) further questioning the power that HR professionals hold to consistently and ethically implement practices to achieve strong outcomes. 


\section{Methodology}

\subsection{Research perspective}

Qualitative research is increasingly being recognized in the research world as it plays an integral part in "understanding the relationship between people and the natural world" (Moon, Brewer, Januchowski-Hartley, Adams and Blackman, 2016, p. 11). This research will use a qualitative approach which fits within the constructivist paradigm as a research philosophy. Cohen \& Manion (1994) describe constructivist approach "as being based on understanding the world of human experiences" (p. 36), hence the focus of this research approach is to build the meaning of the research topic by comprehending the experiences and opinions of the participants. Considering that people have different values and morals, different background and come from differing social systems (O'Leary, 2017), they are likely to deal with issues and dilemmas in various manner. The constructivist approach accepts “complexity, the unknown, diversity, plurality and multiple realities" (O’Leary, 2017, p. 7) which will be beneficial in this project as HR professionals in different organisations are likely to have diverse values and opinions on their profession. Moreover, these values in most cases will be influenced by the organisational context, hence HR professionals engaging and understanding their profession will be "based on their cultural, historical, and social perspectives" (Moon \& Blackman, 2014, p. 6) and thus creating meaning and knowledge through their interaction with other individuals.

\subsection{Personal philosophical perspective}

As discussed, the constructivist approach focuses on human experiences to understand the complexity of the world. As the researcher of this project, I will be viewing the HR professionals as experts in their field, hence focusing on their views and experiences to explore how it shapes the HR profession and their role when it comes to resolving complex ethical issues. Ultimately, the aim is to make sense of these experience and develop knowledge (Schwandt, 1994) to clearly understand the role of HR professionals. As an intermediate HR professional, myself, I am seeking to comprehend how other HR professionals view ethical dilemmas and what methods they use to build an appropriate resolution. Due to this approach, my personal philosophy is strongly aligned with the research philosophy of a constructivist, as I am assuming that "different individuals construct the meaning of the same phenomenon in different ways" (Moon \& Blackman, 2014, p. 6). Therefore, different HR professionals are likely to have different experiences with ethical 
dilemmas and the influence it has on role-conflicts that HR professionals experience. The collection of these various experiences will enable me to discover the meaning of the range of interactions that HR professionals have with the world consisting of other individuals and organisation members, and how they interpret these interactions (Moon \& Blackman, 2014).

\subsection{Participants}

Given the research paradigm, participants for this project were selected as subject matter experts on the HR profession. They were categorized as experts based on their current role as a form of a high-profile HR professional in an ethical organisation and on their strong knowledge about HR and business environment. I fixated on these individuals due to their knowledge and experiences, and their ability to provide their views on the challenges regarding role-conflict that HR professionals are likely to be confronted with in their profession and recommendations that could be useful for those challenges. Due to the nature of the research topic, it was crucial to choose HR professionals in senior positions as they would have likely held a range of positions within HR, hence strongly shaping their view of HR. Considering the type of participants required for this study, they at times were hard to access and book for interviews. Therefore, a snowball sampling method was utilized to identify further participants if required (O’Leary, 2017).

Initially, with the help of my supervisors and her contacts and my own research, 15 organisations were contacted and invited to participate in this research. The 15 organisations were chosen due to their best practices and strong ethical reputation in the market. Due to these characteristics, HR professionals from these organisations were likely to provide significant, rich input on the HR profession and their personal views on working in HR. Though initially 15 organisations were contacted, I was realistic about the fact that not all 15 organisations would be available to participant due to various reasons. The key contacts from each organisation were first sent an email with a letter attached, explaining the project and the reason for inviting them to participate in this project. Due to the top-tier positions of these contacts, it was expected that the response would not be immediate. A follow-up email was sent a week later to determine whether the selected organisations would be interested in participating. Out of the 15,8 responded with a keen interest to participate and appointments were booked to hold interviews at either their place of work or in a meeting room at Victoria Business School. All interviewees held senior HR positions in their organisations and came from a range of different background in terms of their work experience and educational background. The seniority of their role was also reflected in their position titles which ranged 
from HR Business Partner, Chief People Officer to Head of HR and General Manager People and Culture. Furthermore, all participants held a tertiary qualification from a variety of disciplines such as Psychology, Marketing and Anthropology, and worked in various positions before commencing their HR career.

The final sample number of $8 \mathrm{HR}$ professionals was chosen for several reasons. Considering the nature and position of their work, it was assumed that it would take time to book and conduct interviews with the participants even if they have shown keen interest. Though the delays were unforeseeable, I had to ensure that I was flexible with my time to meet the availability of the participants. Furthermore, all interviews were approximately 1 hour and due to the in-depth nature of the interviews, I had to be realistic with regards to their availability. 6 out of 8 interviews were conducted face to face which gave both parties an opportunity to build a good rapport before commencing the interview; 2 interviews were conducted via telephone which was equally valuable, however, the interview was mostly formal, rather than a two-way conversation like the others. The final sample was made up of 3 public sector organisations, 3 private sector organisations, 1 independent consultant working for various organisations and 1 financial service organisation with 6 females and 2 males. The diverse nature of this sample group ensured that the study is provided with an extensive range of perspectives in terms of working in HR and the future of the HR profession.

Below is a summary of the participants which will ensure that participants are easily identified in the findings and analysis section:

\begin{tabular}{|c|c|c|}
\hline Participant & Sector & Gender \\
\hline P1 & Private & Female \\
\hline P2 & Private & Female \\
\hline P3 & Independent & Female \\
\hline P4 & Public & Female \\
\hline P5 & Private & Female \\
\hline P6 & Public & Male \\
\hline P7 & Public & Male \\
\hline P8 & Financial Service & Female \\
\hline
\end{tabular}




\subsection{Data Collection}

The data was collected using semi-structured interviews to accurately explore the HR profession. Considering that the research topic focuses on dealing with ethical issues, it can sometimes be difficult for interviewee to answer questions in a natural manner. Therefore, the interview started with set questions to engage with the interviewee and build a rapport. However, the aim was to have a "flexible structure to allow a natural flow of conversation" (O'Leary, 2017, p. 240) to follow the tangent of the interviewee. This interview structure allowed to gain the data that was intended along with additional data that may have been unexpected (O’Leary, 2017) but provided further insight into the research topic. Before beginning the interviewing process, I conducted a pilot interview with an HR Manager at an education institute to ensure that the questions and the sequence of the questions were appropriately framed for participants to understand and respond. The findings from the pilot interview were purely used as a practice and were not included in the data set. Furthermore, considering the semi-structured nature of the interview, it was possible for interviewees to explore other tangents which may or may not be relevant for the research question. Therefore, I had to be mindful of using appropriate prompts and probes to keep the interview on track but also explore other angles as the interview progressed (O’Leary, 2017).

The interview commenced with an introduction of the topic and an explanation of how and why the research question was created. The participants were reminded that the interview will approximately be one hour, and it will be recorded for it to be transcribed after, giving them the opportunity to review their responses. Once, all the protocols were explained, participants were given a consent form to read and sign. The first section of the interview focused on getting to know the participant, their background in HR and why they chose to have a career in HR. The aim of this section was to ease the participant in the interview and to measure their personality and their initial views of their current position and HR in general. The next part of the interview explored the topic of role-conflict. Before asking the interviewees their experiences with regards to role-conflict, I provided them with the findings that some of the management academics found with regards to the different roles that HR professionals must comply with. Providing interviewees with this background enabled them to think about their current role and what roles they may be undertaking. Once it had been established whether they experience or did not experience role-conflict, the interview went on to the next section relating to ethical or sensitive situations. By identifying a difficult or sensitive situation at work, it allowed the respondents to think about how they resolved the situation while 
thinking about how it affected the role-conflict that they experienced in their roles. The final section of the interview provided the interviewees with the opportunity to bring their views together on role-conflict and ethical situations and deliver their best practice and recommendations for coping with role-conflict in an ethical situation. Considering the structure of the interview, each section had to be covered before moving onto the next section. Therefore, participants were asked to think about role-conflict before thinking about an ethical situation they may have experienced, and then consider best practices for coping with role-conflict in an ethical situation. The recordings were transcribed after each interview, and the transcript was emailed to the interviewee to review and consider any changes.

\subsection{Data Analysis}

The interviews were transcribed within a day after the interview date. This was useful as the content of the interview was still fresh in my mind, hence making the transcribing process more efficient. Once the interview transcript was approved by the participant, the next stage in the research was to look for appropriate themes and analyse the data. "The idea was to systematically drill into the data to build categories and sort it into various themes" (O'Leary, 2017, p. 332) to create meaning. This involved going through each transcript very closely to look for shared ideas to determine the common themes deriving from the interviews. Initially, some of the categories such as 'role-conflict', 'ethical situations' and 'the role of HR' were pre-determined based on the literature review and the research question, hence coding was conducted accordingly. Furthermore, new themes also emerged as the interviews progressed such as the 'future of HR', hence requiring further coding and categorisation. The coding process included colour-coding phrases, sentences and words to determine themes with each colour set for one particular code. In addition to determining themes, keywords were explored through their repetition and the context within which they were used (O'Leary, 2017, p. 333), therefore focusing on the commonality of the words used to comprehend the participants perception and emotions with regards to working in HR and the impact of roleconflict on the HR profession. For example, when asked how they deal with role-conflict when confronted with an ethical situation, words such as 'consistent' and 'fair' were commonly and repeatedly found in most of the interview transcripts. Lastly, some words and phrases were categorised as metaphors as they brought "imagery and empathetic understanding" (O'Leary, 2017, p. 333). For example, expressions such as 'punching bag' and 'walking on fine line' were used to describe the impact of role-conflict or when 
confronted with a complex ethical situation. Once, the coding process was completed, findings were inserted into a matrix to determine the correlation between the themes and each participants' response with regards to those themes.

\subsection{Credibility}

The objective of this research is to explore the HR profession and produce knowledge that other organisations and professionals can rely on (O'Leary, 2017) and implement in their own roles and organisations. To maintain credibility in this research, I ensured that subjectivities were appropriately managed to achieve the "truth value" (Moon et al, 2016). When commencing interviews, it was imperative to be mindful of the fact that it is the "society itself that is being researched" (O'Leary, 2017, p. 57), hence each different perspective had to be respected and appreciated. The aim was to focus on objectivity and to put aside any biases and preconceived ideas (O'Leary, 2017). For example, having worked in HR before, I had the pre-determined knowledge that HR can be focused on processes, hence labelling it as an 'administrative' function within an organisation. Therefore, when I conducted the interviews, I ensured that the participants expressed their views on their own terms, without me providing them with any leading questions (O'Leary, 2017). Furthermore, I managed potential subjectivities by working with my supervisor and utilizing her additional viewpoint of the results and analysis (Moon et al, 2016). The different perspective established that any biases had been avoided. Lastly, providing the participants with the interview notes (Moon et al, 2016) helped me determine if the information shared by the participants had been interpreted and recorded in an accurate manner. 


\section{Findings and Analysis}

The aim of this research was to explore the HR profession and the impact of role-conflict on HR professionals when confronted with an ethical situation. HR is a core function of an organisation, ensuring that the organisation is run smoothly, and the employees act and work in an ethical and productive manner. Due to the current business climate and the emphasis on the ethical work environment, the HR function has been under scrutiny in terms of their role and capability to contribute to the overall business goals. Due to blurring boundaries, the literature suggests that the HR profession needs to think about upskilling and becoming a strategic function rather than staying as an operational function (Lemmergaard, 2009). Considering the research question, the findings and the analysis is focused on three distinct parts; role-conflict, ethical situation and role of HR followed by a summary of the analysis and the main points from the analysis.

\subsection{Role-conflict}

The four roles discussed in the literature; Administrative Expert, Strategic Business Partner, Change Agent and Employee Champion were strongly agreed upon by the participants, however, the degree to which they carry out each role was dependent on their organisation and situational context. Participants claimed that "you have to be able to be all of those four things when it's called upon. So, I don't see that you have to sacrifice any one of them while you are focusing on the other' (P1) and "My view is that you can do all four, and I would say that my job is do all four. And if I am truly going to be successful, I need to figure out how much time I need to spend on each of those areas" (P4). Hence, there is a consensus that the ability to do all four is clearly achievable, however, a degree of prioritisation is strongly required to ensure that all four roles are carried out successfully. Alternatively, there is a belief that role-conflict does exist for HR professionals as according to one participant "you do end up in situations where the lines are blurred" (P7) and another one said "I think the time you often experience role-conflict is when you are in a situation where you might be working with the leadership team and there is also an employee issue you might be dealing with" (P4). Furthermore, one participant used the analogy of a 'punching bag' (P5) to express the feelings of when trying to manage role-conflict.

The concept of role-conflict is significantly visible within the HR profession due to the changing nature of their role and the business environment. Aligned quite concurrently with role theory, it is clear that depending on the situation, HR professionals are required to wear 
multiple hats to meet the numerous demands within the organisation. Hence, the findings suggest that to truly succeed as an HR professional, the profession needs to have the ability to understand their multiple stakeholders and different situations and adjust their behaviour accordingly. However, it is also imperative to note that this may not always be possible as personal values and morals might play an integral role when making decisions. Furthermore, literature claims that the main role-tension tends to be between the strategic business partner role and the employee advocate role (Harrington \& Rayner, 2010), which is aligned with the findings as participants view role-conflict to be more visible when the situation involves two parties who hold differing positions within the organisation.

Considering that the participants are senior HR professionals, it is evident that strategic input for the organisation is one of their main roles and priority as they "like the idea of impacting the organisation with great, fresh ideas and new people" $(P 6)$ and as claimed by $P 3$ "we are about the organisation, and getting the best out of that organisation, and getting an end result that the strategic drivers tell us" therefore supporting the claim made in the literature that the HR profession is becoming more strategic. Furthermore, it is strongly believed that "ultimately, it's about the business" (P5) and as mentioned by one participant "first and foremost, you are there to make sure that the organisation functions smoothly" (P6). In contrast, one participant argued "one of the biggest conflicts that I see for HR professionals, is that it seems HR is there to protect the organisation, in my view, HR is there to promote the well-being and the worker experience of ours staff" (P7) highlighting the issue of roleconflict.

The strategic business partner role of HR professionals has been widely discussed in the literature, as scholars believe that role of HR will be more beneficial if they move toward being strategic (Francis \& Keegan, 2006). The findings suggest that the move to a strategic role is favoured by HR professionals as it provides them with the opportunity to enable the organisation with innovative and strategically driven results. Moreover, though the issue of role-conflict has been highlighted by the participants, it is also strongly evident in the findings that at the end of the day, it is about what is best for the organisation and the outcomes required to enable the organisation to work at their highest potential. The participants' views on this role were similar to what has been said in the literature as it claims that the "focus is on ensuring the overall needs of the organisation" (Lemmergaard, 2009, p. 184). A slightly different perspective was expressed by one participant who focused more on the well-being and experience of the worker. However, it is possible that the well-being of 
the employees can only be achieved if the organisation is performing competently, as that will influence the employee work ethic, productivity, and loyalty.

Though it is clear that to be truly successful, all four roles need to be met by HR professionals; given the position of the participants, the administrative expert function was least executed by the participating HR professionals. One participant said "I personally don't do so much of the administrative expert role, however the team definitely do" $(P 1)$ and another participant expressed "the administrative aspect is probably the area that I prefer not to dabble in too much and that's where I use the skills and strengths of my team" (P2) while another participant, though agreeing that administrative part is often led by the team, said " $I$ don't actually think you can really be effective in your role and get away with some degree of admin no matter what the role” (P4). In comparison to the strategic business partner role, the administrative role though considered important, is often seen to be a task conducted by the team managed by the HR professionals rather than HR professionals themselves.

The sample for this research consisted of HR professionals that are in top-tier roles, hence possibly working very closely with the line managers and the leadership team. The findings suggest that the administrative function of HR is executed by the team, rather than the senior HR professionals themselves which potentially might be the reflection of the sample, as junior HR professionals may still execute this function. Additionally, the nature of the administrative function is to focus on 'operational' activities (Brown, et al, 2017) and 'work processes' (Francis \& Keegan, 2006), hence highlighting that the attention is on the smoothrunning of the organisation. Furthermore, considering the process-driven nature of the role, the literature emphasises that administrative experts can be viewed as a low status position within the organisation (Brown et al, 2017, p. 9). This claim is correlated with the findings as there is a lack of administrative execution from senior-level HR professionals.

Given the nature of the participant's roles, almost all participants were part of projects that involved driving a change of some class within their organisations, hence acting as a change agent. When talking about one of the projects that the participant is part of, they claimed " $m y$ role has become more of a change agent, a coach, a reference point for people who want to bounce ideas or hear ideas" (P2) whilst another participant said "we have done a lot of that (change), both to support changing business strategy and preparing ourselves for the future of work" $(P 1)$. Hence though the role of change agent wasn't explicitly mentioned, it appeared that the interview questions gave the HR professionals an opportunity to really 
think deeply about their roles, the projects they were involved in and how it ultimately impacts the organisation and their own roles.

The role of a change agent simply involves promoting the required change within the organisation and executing it in an efficient manner (Lemmergaard, 2009). The findings suggest that HR professionals are responsible for ensuring that the change is executed in a manner that the leadership team and employees can accept with ease. As expressed by one participant, when conducting a change-related project, HR professionals become a 'reference point' (P2) for employees and the leadership team for them to be able to adapt to the new processes and protocols. Scholars in the literature say that HR is not directly involved in the decision-making of the change required, but more focused on the "delivery of the organisational transformation" (Lemmergaard, 2009, p. 185). Therefore, there is a degree of similarity between the findings and what has been said in the literature, as being a 'reference point' relates to being the HR function that drives the change while ensuring that everyone in the organisation understands the implications of the change.

Lastly, the employee advocate role has traditionally been HR professional's focus, however, with the emerging change, this focus is also changing. As one participant stated, "I actually don't think HR is an employee champion as it still comes back to the organisation" (P3) expressing a strong emphasis on the fact that everything ultimately comes back to the organisation. Further, there is also an understanding that business objectives and the wellbeing of the employees can be balanced, however, it is also where the HR professionals can experience the friction. One participant said "I think the key focus for us is trying to find ways to make sure that the outcome is good for the employee and the business" (P5) and "we bring things back to our people element while trying to get that business objective" (P6), therefore articulating the act of balance, rather than just focusing on one. When looking through an employee perspective lens, $P 2$ claims "I think the staff is always questioning because HR is part of the business and they are part of the leadership team" therefore showing the potential confusion employees may often experience in terms of what HR does.

Organisation employees are required to be engaged and committed in their roles for the organisation to achieve its overall business needs. The role of HR professionals as employee champions has been repeatedly questioned in the findings as it appears that ultimately it is about the business objectives and achieving those objectives. The findings propose that there is an increasing need to achieve a balance between achieving business goals while ensuring 
that the employees are satisfied with the outcome as employees are the driving force for business objectives. This need for balance illustrates that processes and initiatives in relation to employee well-being, employee engagement and retention and development should perhaps be included in the organisation's best practice rather than having a singular HR function of an employee champion. The participants' view on the role of employee champion is considerably different to Ulrich's (1998) view who believes that the HR function should be employee's voice in terms of concerns and recommendations to the leadership team to raise engagement (p. 130). This difference further highlights that the profession has changed, and the focus is increasingly on the organisation, rather than on just on a single stakeholder.

Through the interviews, it has been established that there is a degree of role-conflict that HR professionals experience in their profession given that they are trying to navigate roles which liaise with different stakeholders. Furthermore, due to the nature of their position and as one participant said, "as an exec and a senior leader, I do work for the company" (P2), it is understood, no matter what the situation, essentially it is about the organisation and it's needs. This highlights that the profession's primary goal is to achieve an outcome which is best for all parties to protect the organisation from vulnerability and reputation damage. The participants agree that it is achievable to do all four roles if the HR professionals are skilled in prioritising and analysing any given situation.

\subsection{Ethical Situations}

"HR tread that middle ground" (P6) was a significant theme that derived out of the interview in terms of coping with an ethical situation within an organisation. The next part of the interview with regards to ethical situations provided the participants with an opportunity to consider a sensitive or difficult situation they have experienced. All participants had experienced interpersonal deviance which involved a number of different parties. These involved circumstances such as performance management, bullying, sensitive workplace audit and difficult conversations with the leadership team. There was also a common agreement that the intensity of role-conflict is higher when there is any form of ethics-related issue in an organisation. Participants expressed views such as "if any kind of unacceptable behavior happened at any level of the business, it is absolutely fair to say that it would get harder to deal with, the more senior the person which is why we use the relative independence of HR personnel in interventions" $(P 1)$ and "you are challenging behaviours and so, you actually have to hold yourself accountable for every action, the thing you say, do and respond to" (P2). When discussing the outcome of an ethical situation that the 
organisation leaders decided on and which the participant wasn't comfortable with, they said "it did end up with me choosing to leave that organisation" (P8) illustrating the intensity of role-conflict

Considering the sensitive nature of an ethics-related situation, it appears to be crucial for HR professionals to ensure that their behaviour and actions are aligned with the organisation's objectives. Furthermore, given that they are required to not only manage situations from the organisation's point of view but also from the other parties involved (such as the employees), it is imperative for HR to act in an unbiased manner as they are required to challenge the actions and hold multiple people accountable, which may include members of the leadership team. The findings also suggest that dealing with an ethical situation becomes more challenging when people involved hold high-level positions within the organisation, implying that role-conflict becomes more visible and highly intense. Hence, the act of balance is strongly required to ensure that the decision executed is right for the organisation and for the parties involved. Moreover, literature states that the power HR holds within an organisation is very limited given the various roles they must undertake in addressing different organisational members, which ultimately shapes the decisions they make (Linehan \& O'Brien, 2017), further illustrating the strong need for HR professionals to achieve an unbiased balance when making any decisions that might affect more than one party.

"Grey" was referenced several times in the interviews to illustrate the ambiguity of various situations that HR professionals are often required to operate in. It showed a significant contrast from traditional HR practices, where situations, people and life may have been clearcut. When discussing the severity of punishment for misconduct, one participant said: "it depends on exactly what they have done, because there are some things that are so black and white, but a lot of what we do operates in shades of grey" (P5). The connotation of 'grey' was also related to understanding people and their perspectives, which through the interviews, seems to be one of the main characteristics for working in HR. When examining an ethics-related situation, one participant claimed, "often having someone who is not a lawyer coming in is quite good because they can look at the grey area and think 'well ok, what would a reasonable person think like?"” (P3). As "people are people” (P2), there has been a heightened need for HR professionals to deal with different shades of 'grey' to think outside the box and provide constructive solutions. 
Furthermore, themes of 'consistency' and 'fairness' were strongly projected with regards to coping with ethical situations. The belief was to eliminate any risk that the organisations may be vulnerable to when certain situations arise by ensuring that everything has been done to achieve a fair outcome for the parties involved. Therefore, when working to resolve sensitive issues, P5 said: "you are supposed to exhaust the process before you get to that point of final determination". Other participants said "if you are fair and consistent, then the best you can hope for is respect" (P3), and "you need to know what your parameters are but act with confidence, act with authority and act straightaway, and if the same thing happened 6 months later, make sure you are applying the exact same process" (P1), showing that consistency is highly desired when it comes to coping with ethical situations, as it enables the HR professionals to cover all grounds before the final decision is made. Furthermore, one participant mentioned "when you are investigating, you need to ensure that you are doing the right thing by the person who is complaining, but you also need to do the right thing by the person that needs to respond to that" (P8), showing the importance of considering all viewpoints before delivering the right outcome.

Ethics is a complex phenomenon which at times can be difficult to comprehend. As repeatedly mentioned by the participants, functioning in situations that is not straight forward is part of working in HR. As stated by Lawton et al (2013), unethical behaviour and actions differ from context to context subject to the situation and organisational culture. This view from the literature correlates with the participant's view of knowing how to operate in situations that may be known as grey areas, further showing the need to be able to deal with uncertainty in the everyday organisational context as each situation is likely to be different. In addition to working with ambiguity, the themes of fairness and consistency strongly proposed by the participants show the importance of considering all possible options before deciding on an outcome, particularly in situations that are not clear-cut. This relationship between 'greyness' and 'fairness' highlights the emerging theme of following a robust process and being consistent in that process, to show credibility and duty of care in decision-making. Furthermore, it also shows that HR professionals highly rely on following a process to ensure that the right outcome has been achieved.

Lastly, going back to your own 'personal values and morals' were consistently mentioned in the interviews when combining role-conflict and ethical situations together. This is highly imperative when the situation is sensitive and multiple parties are involved, and as one participant mentioned "you have to understand your own drivers and you have to be honest 
with yourself", (P7) whilst another said "first and foremost, you must ask yourself, 'are you doing the right thing?' because there are times when rules and guidelines don't necessarily serve the purpose of doing the right thing by a human being" (P8) highlighting that if decisions are made that are not aligned with what one truly believes, then the likelihood of making a wrong decision is very high. Similarly, another participant used the analogy of 'head and the heart' to bring 'values' and 'the right thing to do' together and said, "The 'heart' is we care about people and you want a good outcome for the people and the company. But the 'head' says, "what actually occurred", "what are the legal requirements around this and what precisely do we need to do to look after the parties concerned". That then directs your action. But you can apply the heart by being respectful and just generally looking after people” (P1). Therefore, emphasising that HR professionals must be mindful of their actions and personal values by considering all the facts to make a fair decision that is respectful for all parties involved.

The importance of personal values and morals by the participants illustrates the complex human behaviour and situations that HR professionals are required to deal with. There is a consensus that when making a fair and consistent decision, considering personal beliefs and ethical stance along with the situational fact and legal obligations, ensures that the decision is likely to be right as all facts have been considered. This finding could be a little biased, as the sample is made up of senior HR professionals who may hold the power to consider their own values which may or may not align with the organisational beliefs. Therefore, HR professionals who are working at a lower level may not hold this power.

\subsection{Role of HR}

The role of HR has significantly changed in recent times due to various constraints in the business world. Further, it has moved from the operational role to more of a strategic, leadership role. Almost, all interviewees confirmed that, they must do what is best for the organisation, but also consider the impact it will have on the employees and any future organisational design. As participants expressed "there is a lot of talk now about agile workspaces and how to move organisations forward in terms of being able to act to the minute. I think there is a lot of pressure put on the HR function to operate quite quickly, to adapt to certain ways of acting, etc" (P6) and "I am constantly needing to grow and evolve as humankind grows and evolves. So it means that I very much need to be thinking about the people, and how to get the best outcome for both the individual and the organisation (P8), 
showing that the profession must constantly evolve to keep up with the changes in the workplace strategy, business climate and society in general.

Given the nature of this constant evolvement, some of the HR responsibilities have also evolved. In several interviews, participants mentioned that some of the HR tasks are now completed by the line managers, therefore making HR more of a support and advising function to the managers. When discussing the changing role of $\mathrm{HR}$, one participant said "if HR is used where the managers can trust them to coach and to provide them with support along with guiding managers and challenging their thinking in a safe way, then HR is extremely important" (P3) whilst another participant said "HR needs to be advising, coaching, and helping leaders really understand that there is people things that they need to think about" (P4) highlighting a new potential role emerging for HR professionals of a 'coach' or a 'mentor'. Therefore, with line managers taking on more of the HR responsibilities, "there needs to be a lot of coaching and mentoring that needs to happen around them" $(P 6)$ for them to make accurate decisions in a safe and ethical manner. This change contributes to the profession becoming more strategic as the operational tasks are slowly being carried out elsewhere within the organisation.

Further to the emergence of the role of 'mentor' or 'coach', the participants also strongly believed that HR should be sitting alongside the management team due to their ability to provide a strategic view on the people component of the business. Participants shared views such as "my personal view is that HR should be at the top table, and helping to drive the people part of the organisation" (P4) and "I think a good HR person should be able to sit at the management table because their role is to go 'ok that's where we are heading, here are all the bits to get us there from a financial point of view, your marketing, your operations"," (P3). A couple of participants agreed that the move to the leadership table is steadily occurring for HR, however, it still requires recognition to be at the top table in comparison to other functions. They mentioned "there is a huge movement in the sense that we are represented at the leadership table. We can look across lots of multiple angles. There also needs to be a strong partnership between the HR Head and the CE because the CE has the ultimate accountability for the people function" (P2) and "I always find it very bizarre, though most places are doing that now; that sometimes HR is not given a seat at the table when finance is given so much prominence, but actually you need your people to run the business" (P5). 
With the strong emphasis on HR needing to be at the leadership level, it is evident that the HR profession is no longer just operational. The emerging change has also influenced the strategies that HR professionals utilize to resolve role-conflict which is present within the profession. When discussing the need to deal with role-conflict in an ethical situation, one participant said "I would say have a coach, have someone who can say to you "pull your head in here and think about this" or "you are getting too wound up in the detail here", (P7) while other participants mentioned the idea of having an independent body that HR professionals might be able to use for additional advice. They said "what I would naturally do is consult with my peers, but sometimes you actually can't do that because it might involve one of your peers and it might be such a big issue that you don't want to risk it leaking within the organisation" (P2) and "HR field thinks they need to solve it all and yet the business wouldn't hesitate to get external advice, for when they need it. I would like to see HR do more of that (P8), highlighting the increasing need for supporting functions for HR professionals themselves given the complexity of their role. Furthermore, participants also highlighted the need to have a strong understanding of the organisation practices and policies to cope with difficult situations. As one participant said "I think you have to be very clear on the guidelines and recommendations set out in terms of best practice and use that as kind of as the base level. I think when you come from the hard and safe place of knowing the ins and outs of best practice, you are more able to push back and give people clarity around why you do things" (P6) while another mentioned "the standard that you walk past is the standard that you accept" (Pl) showing the influence of policies and the manner of how those policies are carried out on decision-making. Similarly, one participant talked about the importance of organisation culture when dealing with an ethical situation and expressed "I think a lot of that comes down to the culture of the organisation and the level of engagement that we have with our people at multiple levels, hence at the leadership level and also with HR, etc" (P4), illustrating the significant relationship between the HR function and the organisational culture.

Additionally, derived from the interviews were certain individual characteristics that were considered desirable to be effective in the profession. When discussing coping mechanisms for dealing with role-conflict in the profession, one participant said "I think a critical ingredient for any HR person is an understanding of people and a tolerance for people. You also must be very patient and empathy is integral " (P2) and another participant mentioned, "you have to understand people, what drives them and what motivates them, because 
people's displayed behaviour is only a symptom of the underlying attitude" (P7). These findings illustrate the strong weight on 'people' and the need for skills such as tolerance and empathy to truly succeed at being a credible HR professional. From a different perspective, one participant expressed "I often say you don't get into HR to be liked"(P3) whilst another participant said "I think there is a bit more recognition around the fact that being nice is not part of the role description anymore. I think that's definitely a step in the right direction" (P5) further showing the change in the profession by challenging the traditional stereotype around the HR function.

The change to more of a strategic function has provided HR professionals with an opportunity to contribute to the overall business objectives, ensuring that leaders recognize the importance of people as "people are your business" (P2) and as one participant said "without people, most businesses in NZ won't exist, and in most businesses it's their number 1 asset" (P8). However, from an employee and leadership perspective, the change in the role of HR may cause confusion with regards to how they can utilize HR and their expertise. When asked about the perception of HR within organisations, participants articulated views such as "you can follow the process and make sure that it's very fair etc, but it's still seen as a bit of a police function" (P2) and "there is always that joke of 'don't get HR involved, we will never get this resolved' and that they make things more complicated than they need to $b e$ " (P3) highlighting that even when the process is about achieving a fair outcome, there is always some negativity with regards to HR getting involved. Additionally, $P 2$ expressed "with management, I think there is a little frustration at times, because we hold managers to account with policies and practices, and they have the perception 'oh HR is on our case again'”, further emphasising the negative undertone around HR. Alternatively, there is also a belief that how HR is perceived is subject to individuals' own values. When discussing the employee perception of HR, one participant said: "it would very much be dependent on their own values, personal experience and the things that matter to them" (P4) highlighting that to a certain degree, the perception comes down to the individual themselves and what they expect from HR.

The ideas originating out of the findings show a significant evolvement of the profession. In addition to the four roles discussed that HR professionals must comply with, new roles of 'coach' or 'mentor' has emerged in the findings. This appears to be the result of the shifting of the HR responsibilities, where line managers are taking on some of the operational tasks such as recruitment, training, and development. The potential issue with this may be that 
some line managers could have developed into managerial roles through their technical expertise and tenure within the organisation with little or no experience in people management. With increasing change in workforce demographics and way of working, it can be complex for line managers to manage their staff, hence building the role of HR in terms of coaching and mentoring the line managers more imperative than ever. The move to a distributed model of HR also contributes to HR becoming more strategic as they are likely to be working more closely with the line managers and the leadership team. There was also a strong consensus in the findings that HR should be at the leadership table along with the organisation leaders. The understanding is to bring the people-element of the business central to the business purpose, making it noticeable to the organisation leaders that people ultimately run the business. Additionally, the prominence on right practices and policies was also strongly focused on when looking to cope with role-conflict that is visible within the profession. Considering the complexity of the profession and the need to continually operate within the 'grey' area, there was a strong agreement that though robust organisational policies are essential for a high functioning organisation, following the process and ensuring that you live by them is more important. Furthermore, having a mentor or an independent body specifically designed for HR professionals was also seen as an ideal best practice when looking to resolve an ethics-related situation. This is likely to provide the HR professionals with an opportunity to get further guidance from an unbiased party who can provide options from a fresh perspective.

Considering the change to a strategic position, characteristics such as patience, empathy and an understanding and tolerance for people are highly desired to be able to cope with complex situations. Additionally, it has been strongly highlighted that 'being nice' is not needed when working as an HR professional. These characteristics seem to align with the previous theme of being 'fair' and 'consistent' as when dealing with ethical situations, patience and empathy are likely to lead to a fair and consistent outcome. Furthermore, given that HR professionals are required to continuously operate in 'grey' areas, traits such as patience and empathy along with having a tolerance for people show the need to be comfortable and confident when dealing with situations that are ambiguous. Moreover, the perception of HR from various organisation members such as employees and management still seem to be of a 'police function'. There seems to be an ambiance of negativity associated with the profession which might have developed from factors such as the traditional views of HR, the emerging awareness that HR works for the organisation and the process-driven nature they must 
comply within. The main idea that participants heavily emphasised on was that at the end of the day, it comes back to the organisation, hence showing that HR professionals support the organisational leaders. This theme aligns with the social learning theory, suggesting that rolemodelling from the leaders sets the organisation culture, role clarifications, and morale (Brown et al, 2005). Therefore, it appears that the perception of HR within an organisation and the role that they play strategically and operationally is dependent on how the organisational leaders utilize HR and the culture that they promote. Considering this emphasis on organisations, it is imperative for senior leaders to "invest in HR as if it were a business" (Ulrich, 1998, p. 134), which could change the perception of HR, enabling the profession to implement changes that will benefit both the employees and the organisation.

\subsection{Summary}

The HR profession is a complex profession with many challenges, but it's also a profession with plenty of value that will heighten the organisation's reputation and performance. The findings suggest that there are several challenges within the profession that hinder their ability to be productive and perform at the highest standard. A lot of these challenges are due to the different stakeholders they must respond to, navigating situations with unclear outcomes, ensuring that organisation moves forward in a safe and ethical manner along with "market pressures and reduction of trade unions" (Harrington \& Rayner, 2010, p. 5).

Considering the various constraints within the profession, the aim for organisations and HR professionals is to increasingly break traditional stereotypes of the profession to establish their full potential (Ulrich, 1998).

\subsubsection{Main points from the analysis}

Given the complexity of the HR profession, role-conflict, as described in the previous section, is visible within the profession due to HR professionals needing to guide different situations and people while thinking about their own values and morals. Using metaphors such as 'punching bag' to describe the feeling of coping with role-conflict and working through situations involving leadership and an employee, illustrates the intricacy of the HR profession. Alternatively, though role-conflict exists, participants strongly believed that it is feasible to do all four roles. The feasibility is only possible if HR professionals are competent in prioritizing each of these roles as and when required. Additionally, the recognition of having an independent body or a mentor figure that can provide support to HR professionals, particularly in difficult situations illustrates that HR can't solve it all, especially in an organisation that doesn't promote a strong organisational culture. 
The four roles discussed in the literature were recognized by the participants, and it was clear that they undertook all four roles, however at various degrees. The aim of the research was to interview HR professionals in senior positions within organisations, as they were likely to have a wide range of experience in the workforce shaping their views on HR and the business world. Considering this, the participants strongly associated with the strategic business partner role, as their positions required them to work closely with the business side of the organisation rather than the operational side. Furthermore, this was supported by the participant's principle that it was mostly about the organisation and the needs of the organisation.

Traditionally, HR professionals have been regarded as 'administrators' (Ulrich, 1998) and though this stereotype still seems noticeable within organisations, it appears that the seniority of an HR professional shows the degree of administrative duties they undertake. The participants given their position didn't appear to perform the administrative function as it was often handled by their team. Given the 'process-driven' nature of this role, it is not a surprise to know that senior HR professionals are more engaged with the strategic side of the business, ensuring that HR and workplace practices are aligned positively with the business objectives. Therefore, though it was agreed upon by the participants that administrative tasks are part of every role, given their position and their strategic view, it appeared to be a role that was performed at a minimal level. Alternatively, the change agent role was highly identified by the participants, and almost all participants were involved in change projects with regards to increasing engagement and visibility of organisational values. It seems to be a role that is embedded within their current roles, hence ensuring that change is executed in an efficient manner. Considering that the change is usually initiated by the leadership team, HR professionals are responsible for delivering that change, therefore bringing in the human aspect and the technical aspect of change together. As Ulrich's suggests (1998) "this is one of the most significant roles HR can play" (p. 131) due to their drive for the process.

Employee engagement and retention is imperative in today's business environment due to the changing demands of the business environment. The employee advocate role for HR has significantly changed as the emphasis is increasingly on ensuring that the business objectives are aligned with the employee element. HR professionals chosen for this research strongly expressed that ultimately, it all comes back to the organisation. Hence, for HR, there needs to be a degree of balancing act as the attained outcome should be good for both the organisation 
and the employee. This balancing act is significantly required in organisations as the way employees feel about the organisation is effectively a reflection of the organisation itself.

The expectation to comply with the four roles show that HR professionals are expected to wear multiple hats to cover a broad range of responsibilities. As established, the combination of the above roles can cause friction particularly when there are different parties involved. Considering this tension, there is a general agreement from the participants that tension seems to rise when there is an ethical situation within the organisation. Therefore, given their power within organisations, the act of balancing becomes imperative when dealing with sensitive situations. The act of balance further ensures that decisions are made after all processes have been followed thoroughly and reliably. Foote et al (1999) identified "flexibility, creativity and innovation" (p. 93) amongst others as some of the essential competencies for HR professionals, thus showing the ever-changing face of the profession, especially in times of uncertainty and unethical behaviour. These competencies correlate with the views expressed by the participants, who highlighted that operating in shades of grey is now part of the HR function, hence showing the ambiguity in the profession which may require flexible, creative and innovative solutions. Furthermore, HR professionals emphasised being fair and consistent when making decisions because as established, HR relies strongly on following processes to ensure that the proper result has been achieved for any parties involved. Therefore, there is a strong correlation between operating in a grey, ambiguous situation and being fair and consistent when looking to resolve a sensitive situation. It is however essential to consider that as human beings, HR professionals will have their own morals and values with regards to an ethical stance. According to the participants, in addition to being fair and consistent, personal values play a huge role in determining what the right thing to do is, particularly in situations that are not clear. The relationship between 'grey', 'fair and consistent' and 'personal values' illustrates a powerful combination of ensuring that a right outcome has been accomplished, to make sure that the organisation is not exposed to any vulnerability. In addition to ensuring that strong policies and practices are in place within the organisation, executing the policies and living by them is also firmly required to demonstrate the severity of the outcome if misconduct occurs. Furthermore, living by the policies also ensures that there is a strong degree of consistency when resolving ethics-related issues, which consequently influences the perception that organisational members have of HR. In an era of increased globalisation and technological advancement, it is imperative for organisations to "increase their ability to learn, collaborate and adapt, and manage diversity, 
complexities and uncertainty" (Ulrich, 1998, p. 126). Therefore, HR along with the organisational leaders need to ensure that they are ready to meet the demands by working together in a collaborative manner. With many of the HR responsibilities managed by the line managers, and ensuring that organisational leaders understand the people side of the business, HR professionals are now expected to 'coach' or 'mentor' various stakeholders in the organisation to ensure that anything related to people is dealt with in an ethical and safe manner. This transformation is beneficial for HR professionals as it challenges the traditional role of HR which is focused on processes and operational tasks. When it comes to the perception of HR within organisations, it appears that people from various role backgrounds are still learning the value of the HR function. Considering that the changing nature of the HR profession is gradual, employees still perceive HR with a negative tone due to their drive for a process, complying by organisational policies and conducting administration work. According to the findings and the literature, norms and cultural values within the organisation comes down to the way the organisational leaders behave and model those behaviours. Therefore, the way HR is utilized within organisations ultimately is a result of what organisation perceives to be important and the values that they promote, which will likely influence the perception organisational members have of HR and their role.

There are several similarities and differences between the main analysis and what has been claimed in the literature. Scholars such as Ulrich (1997) and Caldwell (2003) have distinctly stated in the literature for the last few decades, that HR professionals should be undertaking various roles to meet the demands within organisations. There is a strong correlation between the claims in the literature and the participant's views as they all agreed that they undertake the four roles but the difference, however, is their ability to prioritise each of the roles. The ability to prioritise is aligned with the Lemmergaard's (2009) argument who claims that a 'best fit' rather than 'best practice' must be achieved between the HR strategies and the organisation goals. The need for 'best fit' ensures that HR professionals think about each individual situation before deciding on what role to take on. Furthermore, the role of leadership within an organisation ultimately sets the role of the HR function within the organisation. As Nubert et al (2009) claim, leadership within an organisation influences employee's perception toward their work and the over-all organisation values along with the organisational culture, something that aligns with the findings of this study. Therefore, the way organisation leaders promote the culture and outline the purpose of the organisation influences the role of HR and the way it's developed within the organisation. 
Alternatively, though it has been established that HR support the organisation, the findings suggest that in an ethical situation, HR professional's values and morals play an essential role during the decision-making process. Linehan \& O'Brien (2017) claim that in an ethical situation, HR professionals struggle to make the right decision as they are required to compete with their own morals with regards to the situation, and what the right thing to do is. This claim differs from the participant's view as they strongly believed that to resolve an ethics-based issue, HR professionals should consider their own values and motivators along with the legal aspects and the facts of the situation to make a decision that is fair and accurate. From a moral development theory perspective, considering their personal values and morals show how HR professionals' reason about an ethical situation. Alternatively, considering their personal values, in addition to legal requirements aligns with the postconventional stage of moral development as according to literature it reflects on "justice, individual rights, and well-being" (Jordan et al, 2013, p.665).

In addition to the similarities and differences between the literature and participant's views, new findings have also emerged illustrating further changes within the HR profession. The role of 'coach' or 'mentor' has developed with HR professionals acting as an advising function to the management and employees. The participants' perspective strongly shows that being a coach or a mentor in relation to people-related issues is highly essential to ensure that safe and ethical protocols are present in the organisation. However, the findings also show that this new role will only evolve further if organisation leaders and the business environment use this function to their advantage. Hence, as discussed previously, it all comes back to the organisation culture and structure. With the HR profession developing, specific characteristics such as empathy, patience, and tolerance illustrate the need to resolve situations which at times can be complex. Furthermore, participants often mentioned the ability to operate in 'grey', hence showing an association with characteristics such as empathy, patience, and tolerance to succeed as an HR professional as it will likely help resolve an issue that is not clear-cut. The desired characteristics and the emergence of a new role increasingly highlight that the HR profession is changing due to the intricacies of various organisation situations and people. Considering that the desired traits are strongly peoplerelated, HR professionals can likely influence organisation leaders to think about the peopleelement when any decisions are undertaken from a strategic lens. This illustrates the importance of HR sitting with the management team at the top table as they can look at things from different perspectives and ensure that it aligns well with the overall business goals. 


\subsubsection{Summary of the Analysis}

HR professionals are increasingly expected to find the stability between organisational needs and the intricacies of human beings. To achieve a thriving business, the right people with the right skills and behaviours are required as with no people, there is no business. In the space of MeToo movement, increasing need for gender equality, globalisation and changing workforce demographics, HR is under constant scrutiny with regards to their ability to work within a challenging business climate and the value they can contribute to the organisation to enhance its performance. With organisations aiming to make HR function more strategic, there are a new set of challenges and opportunities for both the HR professionals and organisational leaders to ensure that the organisation's business objectives are met in an ethical and reputable way.

The role-conflict experienced by HR professionals hugely impacts their behaviour and the way they cope with situations that are of a sensitive or difficult nature. Though all four roles are extremely important within the profession, it is also the reason why HR professionals often tread in ambiguity. Furthermore, the change to more of a strategic position has provided HR professionals to work alongside business leaders to enable them to see from a people lens rather than just the strategic lens, ensuring that organisational needs consider the people element of the business. Considering that HR professionals are increasingly working more closely with the business leaders, they are required to be fair and consistent in their decisionmaking in every situation possible. One of the main ideas from the analysis of the finding is that everything comes back to the organisation, hence HR professionals, first and foremost work for the organisation ensuring they are safe and protected with every decision they make.

The strong prominence on supporting the organisation leaders illustrates the need for organisations to utilize HR to its highest potential and use its value to their advantage. There needs to be a two-way collaboration between the organisation leaders and the HR function to address issues in a manner that will get the best outcome. Furthermore, as stated in the literature, perceived organisation support (POS) can provide clarity to organisation members with regards to their roles, but also whether the work that they contribute to the business goals is valued and recognised (Bush, 2018). Hence, though still coping with the demands of role-conflict, the analysis ultimately suggests that HR profession is focused more on the strategic drivers to develop practices that will prove advantageous for the employees and the organisational needs. Moreover, given that HR often tread in the middle, especially in situations where there is a visibility of power imbalance, it is hence equally important to be 
mindful that HR professionals themselves at times might require some sort of support to get through a complicated situation.

Given the changing nature of business and the influence of factors such as artificial intelligence, millennials in the workforce and climate change, ethics has become a significant feature within the workplace context. With increasing competition, organisations are working at a faster pace to meet these demands, hence making ethical behaviour more prominent than ever (Rayner \& Harrington, 2010). As functioning in the 'grey' area is part of working within the HR profession, ethics can sometimes be compromised due to the ambiguity that HR professionals must work in. The analysis shows that HR professionals always strive to do the right thing by engaging in a fair process, hence resulting in an outcome that will be fair for all parties involved. Along with the continued emphasis on being fair and consistent, going back to personal values and morals indicate the HR professional's strong motivation to provide an outcome which has been systematically evaluated in every situation. There is likely to be occasional friction between personal values and doing what's best for the organisation, hence further strengthening the argument of having a reasonable and thorough conflict resolution process. The desired characteristics for HR professionals such as being patient and empathetic evidently illustrates that the profession aims to take the right course of action by analysing and understanding every side of the story. From the analysis, it has been established that HR is an important function of an organisation given its role in strategy, change, advising and looking after the well-being of the employee. It appears to be a function that looks after the overall organisation needs which is a significant feature that is different to other organisation functions. Considering HR professional's motivation to work in a fair and efficient manner, the analysis further indicate that HR should be seated at the management level as they can provide an over-all perspective from all the different angles within the organisation. 


\section{Discussion \& Conclusion}

\subsection{Summary}

This thesis has explored the role of HR in organisations, especially when there is a presence of unethical behaviour or serious misconduct, and ultimately what this means for the future of HR. Furthermore, the research also focused on organisations who strived to achieve an ethical culture to comprehend what they were doing differently. With the turbulent business climate increasingly becoming the new norm (Van der Wal, 2017), some of New Zealand top tier firms in the legal and political profession have had certain misconduct behaviour that led to organisation's HR function looking the other way (Newsroom, 2018; Tett, 2019), illustrating the growing question of what the role of HR is when things go wrong. HR professionals are often required to undertake four key roles; administrative expert, strategic business partner, employee champion and change agent. Considering this, it's been assumed in the literature that there is a strong degree of role-conflict within the profession as they are expected to deal with multiple stakeholders and competing demands (Ulrich, 1997). This study through 8 semi-structured interviews with top tier HR professionals working in organisations known for their ethical stance shows that role-conflict is recognized within the HR profession, however it is not regarded as a strong issue due to the belief that it is possible to do all four roles, if there is capacity for prioritisation and teamwork. Furthermore, it is evident that role-conflict intensifies when there is an unethical situation (Linehan \& O'Brien, 2017), but respondents believe that a fair and consistent process will diminish any biases and provide the organisation and all parties involved with justice and a satisfying outcome. Finally, this research shows that the way HR is developed in an organisation is highly subject to the culture, norms, and beliefs that are constructed by the leadership team which consequently influences the degree of role-conflict experienced by HR professionals, especially in ethical situations. Therefore, demonstrating a significant correlation between HR and the organisation culture, and the impact it has on ethics and the changing future of work. This chapter discusses the main findings of the research, along with what organisations and HR professionals should be doing for the HR profession to be successful in coping with role-conflict while creating and sustaining an ethical culture within an organisation.

\subsection{Discussion}

It is evident that due to the transforming business climate, organisation ethics is more important than ever. For example, one of New Zealand's top law firms has been under 
significant scrutiny for failing to address allegations with regards to a series of serious misconduct within the organisation (Newsroom, 2018). In addition to a strong reputation damage, the HR function within the organisation was highly criticised for their lack of knowledge and execution in relation to resolving the misconduct in an efficient and ethical manner. Considering this, organisations all around New Zealand have been encouraged to revisit their code of ethics, clarify the organisation's purpose and ensure that employees are working in a safe environment. Additionally, it has also raised questions about the role of HR in organisations, specifically in situations that are not clear-cut. The research question of this thesis was determined to explore the role of HR, to understand their position with regards to their influencing power in organisations, particularly in situations which involve unethical activity. Therefore, ultimately defining the profession's future in an era where unethical behaviour is more easily recognisable, but also no longer tolerated. In addition to the exploration of the HR profession, another significant feature of this thesis was the relationship between HR and ethics, hence examining the influence and role of HR in an ethical situation and the power they hold within organisations to establish an ethical culture.

Scholars such as Ulrich (1998) have stated that HR professionals should be complying four distinct roles due to the changing nature of the profession and the over-all organisation climate. As discussed, Strategic Business Partner, Change Agent, Administrative Expert, and Employee Champion have been regarded as four main roles that HR professionals should be undertaking within organisations in order to be truly effective within the organisation. Moreover, Ulrich along with several of his colleagues has provided updated variations of these roles, but the main theme still seems to be strategic versus operational HR (Lemmargaard, 2009). As a result of the differing roles, scholars further argue that roleconflict is visible within the profession as it presents challenges with regards to dealing with different organisational stakeholders who may be in contradiction of each other (Harrington \& Rayner, 2010), for example; role of strategic business partner versus the role of employee champion. This study found that HR professionals recognize the four roles and tensions it can cause, however in contrary to what was said in the literature, role-conflict though visible in the profession, wasn't seen as strong of an issue by the participants. There was a general belief that in order to be an effective HR professional, one should really be doing all the four roles to a certain extent. The reason for this finding could be the sample chosen for this study as they were HR professionals in very high-level roles, where they had the opportunity to influence decision-making and work closely with the organisation leaders. Therefore, it is 
possible that due to their position in the organisation, the quality of relationship they had with the organisational leaders and the fact that the chosen organisations are known for their ethical culture contributed to role-conflict being manageable. This strongly associates to the arguments of Chiu, Yeh \& Huang (2015) and Bush (2018) who state that adequate support within an organisation can lessen the effects of role-conflict and enable the professionals to recognize that their work is valued by the organisation. Considering this conclusion, it is possible that role-conflict is more strongly experienced lower in the profession, as there is likely to be a lack of power to influence or make decisions and have minimal interaction with the organisational leaders. Furthermore, role-conflict is also likely to be enhanced in organisations that are less ethical with minimal efforts to strive for an ethical stance. Additionally, as seen in the findings, the administrative expert role was least executed by the participants, further showing the influence of their position, as it appears to be more aligned with the strategic HR role rather than the operational HR role. Hence, operating in a strategic role provides HR professionals with the opportunity to work with business leaders, which ultimately would lower the impact of role-conflict.

Considering that role-conflict seems to lessen for HR professionals if there is adequate support from business leaders, it appears that organisational culture has a significant impact on the HR function. Hence, illustrating a strong relation to social learning theory which argues that organisation leaders play an imperative role in providing guidance with regards to organisation culture, role clarifications and ethical expectations (Neubert et al, 2009). In addition to this, the findings of this study show that the role-conflict tends to be higher and intense in an ethical situation, particularly if there are two parties involved with differing organisational power. One of the main messages that derived out of the interviews was that HR professionals are expected to operate in ambiguous situations which is now increasingly becoming part of working in HR. Given the increasing need to work in situations that are not clear-cut, following a fair and consistent process was heavily emphasised on, showing the need to look at all possible options from a range of perspectives to reach an outcome that is not biased. However, a fair and consistent process can only be followed if there are formal policies and code of conduct in place within organisations, which can be a result of the behaviours modelled by the leadership team, and their power to encourage that ultimately shapes the organisation culture.

The findings from this study can shed some light on the case of New Zealand's leading law firm who failed significantly to adequately deal with some serious allegations. It has been 
articulated that the HR function was under strong scrutiny as they "lacked appropriate expertise to deal with the incidents and a number of critical steps in the process were missed" (Bazley, 2018, p. 27). However, Bazley (2018) also reported that "firm lacks or has inadequate policies and systems in a number of relevant areas" (p. 71). For example, it was noted that there was a 'harassment at workplace' policy within the organisation, however when the incidents took place, the policy wasn't followed (Bazley, 2018), therefore not only showing the lack of expertise from $\mathrm{HR}$, but also the inadequate guidance from the senior leaders of the firm, highlighting the absence of ethical leadership. Furthermore, a certain culture was established within the organisation consisting of "poor people management, 'blokey' culture, power imbalance, and a lack of a 'speak-out' culture” (Bazley, 2018, p. 81) influencing employee behaviour and work ethic. Considering the culture, the HR function possibly may have been embedded within the culture, restricting their power to influence and making them ineffective as a people function within the organisation. This shows the connection with the social learning theory, as modelled unethical behaviour by the firm's leaders ultimately contribute to the over-all organisation culture (Brown et al, 2005). Additionally, as shown in this thesis, following a formal process in a fair and consistent manner is strongly needed when dealing with an ethical situation, however, in this law firm, HR function's ability to follow a thorough process was hindered as there were no adequate policies and standards in place for them to follow.

The nature of following a robust process strongly aligns with Ulrich's (1998) thoughts where he implies, "HR should not be defined by what it does but by what it delivers" (p. 124), therefore diminishing the 'policy police' (Ulrich, 1998) stereotype and focusing on delivering results. Linehan \& O'Brien (2017) also argue that decision-making in ethical situations becomes less than accurate for HR professionals as they are conflicted between their own morals and the right thing to do for the organisation (p. 765). This argument didn't align with this study as it was found that considering their own morals and personal values along with organisational needs provided HR professionals with a further clarity with regards to achieving the best outcome in an ethical situation. The emphasis on considering personal values and morals highlight the idea that to lead an ethics-related situation, one should have their own ethical morals, agreeing with Trevino et al (2000) who say that one must be a moral person and a moral manager to achieve a strong ethical leadership. Therefore, if an HR professional doesn't show the right characteristics and behaviours, it is then likely to have an 
flow-on effect on their actions such as modelling those characteristics and behaviours in an ethical situation, ultimately leading to an ineffective outcome.

The role of HR is increasingly becoming imperative for organisations considering the changing business climate, market pressure and aligning business objectives with the peopleelement of the business. It is progressively evident that the HR profession is changing and is focused on becoming strategic than operational to contribute to business needs more effectively (Kuiper \& Giurge, 2017; Brown et al, 2017). In this study, it is shown that some of the operational HR tasks are gradually moved to the line managers as "line managers work closely with their employees and when they assume HR responsibilities this can positively influence employee motivation, commitment, and performance" (Khan, 2012, p. 6). This leaves HR professionals to act as a coach and "offer support and specialist advice" (Khan, 2012, p. 15) to the managers in relation to HR responsibilities such as recruitment and employee engagement, further contributing to the strategic formation of the profession. This is also supported by Kuiper \& Giurge (2017) who say that operational HR role is no longer the leading driver for organisation performance, highlighting the need for HR professionals to become more strategically ambitious. Considering the emphasis on the strategic HR role, there was a strong belief among interviewees that HR should be seated at the management table along with the business leaders as this would expose them to influence decision-making and promote a people-driven perspective. The combination of following a fair and thorough process and displaying characteristics such as patience, empathy, and tolerance of people illustrates HR professional's drive to do the right thing by considering all possible outcomes, but also enables them to bring in a different viewpoint which may not be thought of in the first instance by the business leaders. Furthermore, having a seat at the management table will allow the HR professionals to gain knowledge from the leadership team about the expectations from the HR function (Newell, 2017) which will accordingly influence the organisation code of ethics, policies, and strategies for employee engagement and retention.

With HR developing into a strategic function, organisation leaders need to understand the value that HR can bring to the organisation (Khan, 2012). Hence for the HR profession to be transformed into an effective player within the organisation, the organisation leaders should become HR champion themselves (Ulrich, 1998, p. 126). The chosen HR experts for this study were all from organisations who had an organisation culture that continually worked to improve their ethical stance and a leadership team that worked closely with HR to develop specific practices and policies. Therefore, this study found that a collaboration between the 
organisation leaders and HR ensures that HR thrive in their role and that the structure of an organisation strongly influences the HR function. Considering this, it is increasingly likely that HR will be an unsuccessful function if the culture of the organisation doesn't allow for it to thrive. In the case of the bullying allegations at the New Zealand Parliament Services, the organisation culture was a "corporate hierarchy and dictatorial in which MPs dismissed the workplace code of conduct as unnecessarily prescriptive or overly politically correct" (Vance, 2019), evidently leaving very minimal power and options for HR to implement policies given that the basic requirement of code of conduct wasn't regarded as a priority. Additionally, following the serious allegations around senior lawyers at the New Zealand law firm, the firm's head of HR left the organisation (Newsroom, 2018) further showing HR's restricted power when there is a lack of ethical leadership and culture that normalises unethical behaviour. For HR professionals operating in an unethical organisation, gaining a seat at the management table is likely to be challenging considering that leaders of such organisations are likely to be unaware of the value of HR and the people-element of the business. Therefore, a degree of accountability with regards to the people of the business is with the $\mathrm{CEO}$, showing that CEs need to be aware that the world is evolving along with influencing the business climate where corporate and social responsibility cannot just be assigned to HR (Tett, 2019).

Organisational culture in today's era is more imperative than ever due to the increasing changes in the world and society expectations, which consequently influences the business climate and leadership expectations. Considering the future of work, "there seems to be a growing recognition that operating in an era of continuous complexity and turbulence is the normal state of affairs rather than the exception" (Van der Wal, 2017, p. 39). These turbulent changes in the traditional sense are things such as natural disasters, terrorist attacks, and economic recession, however, it is increasingly becoming a norm for the 'tiny things' (Van der Wal, 2017) such as changes in the individual values and flexibility in work that contribute strongly to the future business climate. One of the key changes in the workforce is the increasing number of millennials that are entering the market, who according to several scholars hold significantly different values than the previous generations (Canedo, Grace, Graen \& Johnson 2017; Van der Wal, 2017). Also, known as generation me (Van der Wal, 2017), millennials are known for being confident, achieving work-life balance, valuing diverse teams and contributing to the organisation goals (Canedo et al, 2017) along with being stereotypes such as privileged, lazy and self-centred (Van der Wal, 2017), illustrating a 
generation cohort that is likely to challenge the traditional work ethic and provide a new perspective on managerial expectations and work environment. Furthermore, the rise of globalisation through the development of artificial intelligence, merging of real and online world through social media and extraordinary speed of transportation (Kaeser, 2019) has not only made processes more efficient and effective for organisations, but it has also "raised the bar for leadership, where leaders stand up for global openness, fair competition and social cohesiveness by taking a clear ethical stance" (Kaeser, 2019) rather than just focusing on organisation and economic outcomes. The 2018 better future report also illustrates the changing nature of business where businesses are progressively expected to be 'kinder' with $86 \%$ of people agreeing with the statement "it is important for me to work for a company that is socially and environmentally responsible" (Colmar Brunton, 2018). Therefore, with the countless significant changes in the workplace, HR's role is likely to be more significant than ever as organisation leaders will need thorough guiding and coaching in relation to achieving an organisation culture that thrives in the turbulent world but remains ethical at the same time.

Emmanuel Lulin, Chief Ethics Officer at L'Oreal quoted in Financial Review said "if we want to measure the success of an organisation, we should look not only at the financial success and what is in the statutory accounts, but we should look at another set of accounts made of trust" (Mitchell, 2019), illustrating the growing importance of ethics in the workplace to achieve a success-driven organisation. Given the growing appreciation of HR gaining a seat at the management table, particularly when ethical leadership and ethical organisation culture is highly substantial, HR practitioners will need to be perceived as credible to obtain this management seat. Therefore, along with being a moral person and a moral manager (Trevino et al, 2000) and having the skills to reason through and resolve ethical issues, it is equally possible that HR professionals will need to upskill and gain another level of training to be perceived as strong strategic people players while continually striving to meet the business objectives. For example, Kryscynski, Reeves, Stice-Lusvardi, Ulrich \& Russell (2018) believe that "analytical ability might be a key skill for HR professionals in order to operate as a powerful strategic HR business partner” (p. 715). Considering the powerful influence of technology and data on organisations, HR professionals may have to investigate the main challenges that the business is confronted with and provide supporting analytical data to determine the appropriate results to the challenges (Kryscynski et al, 2018). For example, with the changing demographic trends in the 
workplace, HR professionals might need to examine the attraction and retention rates of millennials and forecast future trends to design appropriate recruitment and career development plans. Additionally, with the increase in artificial intelligence, HR professionals may need to analyse the correlation between the growth in automation and the current business objectives, to successfully implement a training programme for existing employees to ensure that they are fittingly upskilled to utilize and benefit from artificial intelligence (Taulli, 2019). Furthermore, the analytical skills are even more imperative within the profession due to the gradual transfer of operational HR tasks to the line managers, providing HR professionals with the capacity to work on the strategic matters. Considering that the HR profession is traditionally known for administrative work, it however hinders their ability to gain a seat on the management table. However, with the adoption of "evidence-based methods that validate HR decisions" (Kryscynski et al, 2018, p. 716), HR professionals will have an opportunity to be on an equal outlook as the organisation leaders, rather than encouraging the leaders to take an action (Mullin, 2018). Additionally, given the power of globalisation and generational changes, diversity within organisations will need to be managed innovatively to enable the organisations to succeed in the long run. Mullin (2018) argues that having an HR expert director on an organisation board will enhance the organisation's workplace diversity management due to their people-oriented skills. The study illustrates the evolvement of the HR profession to not just to a strategic position, but to a higher level of being a people expert on a board. This is likely to be challenging to achieve, considering the traditional perception that HR is still associated with and their continuous struggle to be known as a competent strategic adviser.

\subsection{Limitations}

This thesis has provided an in-depth look into the HR profession, the influence of roleconflict on the HR professionals, and their coping mechanisms with regards to this roleconflict, particularly when there is an ethical situation in an organisation. There is a clear understanding that the profession is moving toward a strategic position, providing them with numerous opportunities to contribute to the overall business objectives. Furthermore, considering the perception of HR from various organisation members and the different expectations, the move to a strategic position and gaining a seat at the management table is still a gradual process within organisations. But, like any other study, there are several limitations in this research that also needs to be assessed. 
The small sample size of senior HR experts may have contributed to this sample being biased. The participants from all 8 interviews held senior HR positions in various organisations where they work closely with the business leaders, hence they placed an extensive emphasis on the HR Strategic Business Partner role, as they were more aligned with the top leadership team. Due to the alignment with the top leadership team, the participants did recognize role-conflict to be an issue, however, it wasn't as strongly expressed as it was in the literature. Though the sample was chosen deliberately to ensure that the participants had a range of experience in HR which would make them strong subjectmatter experts, it, however, lacks viewpoints of HR professionals who are currently working in low to mid-level HR roles. There may be a possibility that junior to mid-level HR professionals working in specialist and generalist roles, may experience role-conflict at a greater extent due to the lack of authority with regards to decision-making and power imbalance. Furthermore, considering the characteristics of the sample, the HR profession was viewed from one perspective of HR professionals from organisations that were known for their inclusive and reputable culture, hence focusing only on the 'champions'. The reason for choosing such 'champion' organisations was due to their commitment to making a change, along with the organisation leaders having a strong awareness and value of the HR function and their contribution to the overall business strategy. Furthermore, the 'champion' organisations were also chosen because this thesis wanted to comprehend what these organisations were doing differently regarding their best practices, in comparison to average organisations. Therefore, selecting a diverse, bigger sample number made up of different organisation members such as junior to mid-level employees working in a variety of roles, members of the leadership team such as the marketing director and finance director in these 'champion' organisations will provide a broader perspective of the HR function, and the influence it has on their roles, the overall organisation needs and how it shapes the HR profession. Additionally, with the role of 'mentor' emerging within the HR profession, it is unclear at this stage how well it would fit in with the four already established roles. As seen in the suggested Figure 1, the four roles are connected in an ethical organisation, therefore further research is proposed to investigate the role of 'mentor' and its integration with the other roles in within the organisation.

In this study, the interview process was of a semi-structured format to enable a natural flow of conversation (O’Leary, 2017), allowing participants to critically think about each question and provide their view on the subject. The interview protocol was designed with several 
specific questions relating to role-conflict, ethical dilemma, and best practices, to ensure specific data was collected and any additional tangents were explored as the interview progressed. The questions were prepared through the guidance of the literature, my own personal experience in HR and my supervisor's thoughts about HR and ethics. However, considering the broad nature of the three topics, it is possible that some questions with regards to each of the topics may not have been considered. For example, most of the participants discussed various degrees of interpersonal deviance as examples of ethical dilemma, however organisational deviance such as fraud and theft weren't highlighted, which consequently may have provided another perspective in terms of coping with role-conflict and the ultimate influence on the profession. Furthermore, each section of the interview was commenced with a background of the concepts and the claims made in the literature, however, there is a possibility that the questions were interpreted differently than what it was meant to be implying. For example, when discussing role-conflict in organisations, several participants interpreted role-conflict as dealing with a conflict in the organisation, rather than conflict that arises from role expectations from different members of the organisation. This was minimized by providing participants with an example of role-conflict by distinguishing the expectations of being an employee advocate and a strategic business partner at the same time, therefore liaising with different stakeholder demands, which may ultimately lead to friction between the two roles. Lastly, the data was analysed by categorisation of codes and determining patterns between the codes and the categories, showing researcher bias as I interpreted the results which might portray a generalised outlook of the HR profession.

\subsection{Implications}

\subsubsection{Theoretical Implications}

This thesis has added a new perspective to the literature with regards to the role-conflict felt in the HR profession, but also the complex relationship between HR and ethics. Given the claims in the literature, it was assumed that there is a strong role-conflict in the profession considering HR practitioners are expected to undertake various roles; strategic business partner, employee champion and change agent (Ulrich, 1997; Caldwell, 2003). This thesis adds to the literature by showing that role-conflict is recognized as a visible issue within the profession, however, it is not regarded as strong issue considering that there was a general belief that the profession is gradually and steadily moving toward being strategic and working to align HR practices with the overall business objectives. As discussed in 5.3, this result might be an influence of the chosen sample, as all participants were in very senior HR 
positions, working merely toward strategic goals while providing guidance to the line managers on operational HR matters. Furthermore, given their high position within the organisation, there appeared to be an overlap of the four roles, hence making it achievable for the HR professionals to undertake the assigned roles successfully (see Figure 1). Therefore, future research should explore role-conflict in the HR profession in a diverse sample consisting of HR professionals in a range of positions.

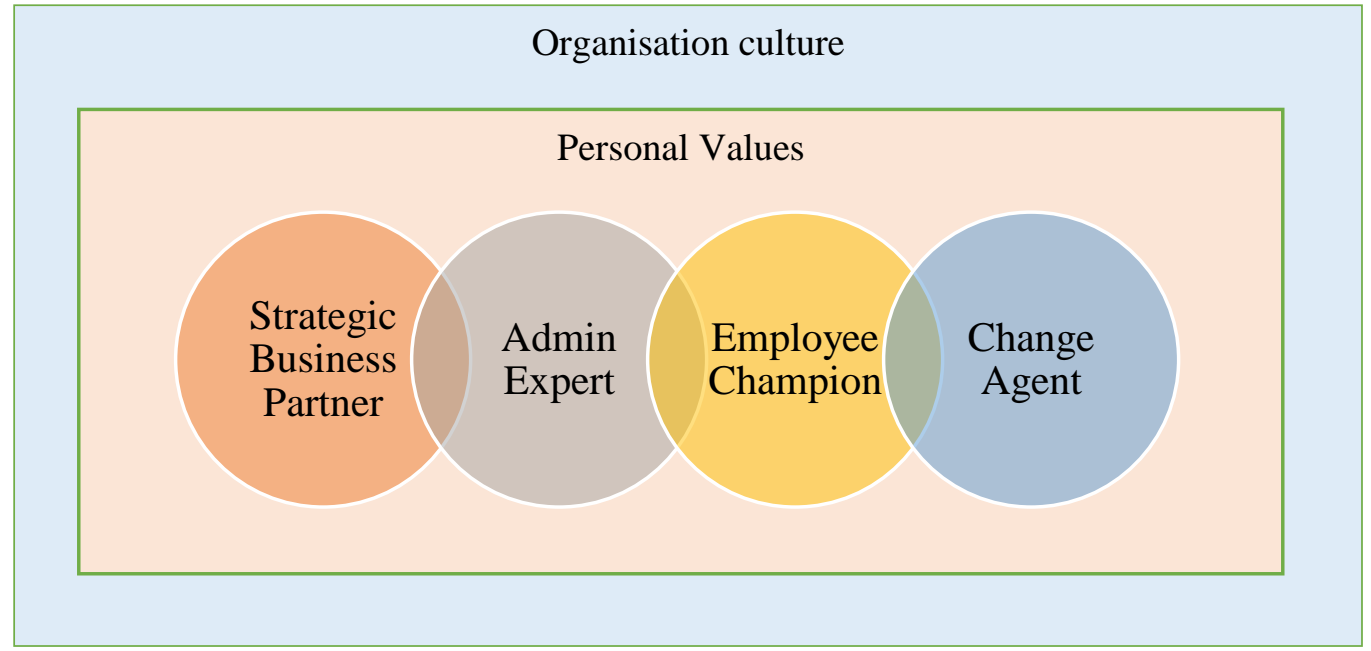

Figure 1 HR function in an ethical organisation

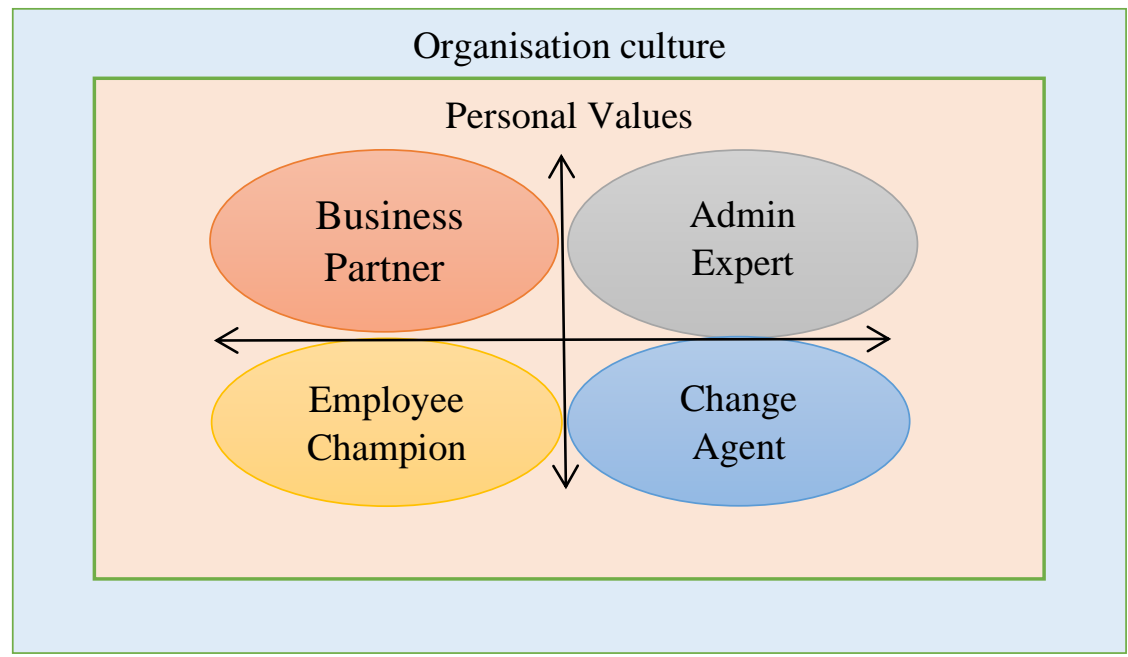

Figure 2 HR function in an unethical organisation

Considering the increasing move to a strategic position, this thesis also suggests an emerging role of 'coach' or a 'mentor' for HR professionals, further adding to Ulrich's models. This is a result of the increasing delegation of some of the HR responsibilities to the line managers who may be inexperienced in people management, hence utilizing HR for guidance and support. Therefore, while working on strategic goals, HR professionals still need to indirectly 
undertake the operational role to ensure that line managers are operating the assigned HR responsibilities in a safe, appropriate and ethical manner. Even though Kuiper \& George (2017) and Brown et al (2017) concluded that the strategic business partner role added more value to the organisation than the operational HR role, given the role of 'coach' and 'mentor' to the line managers, it is equally beneficial for HR professionals to still be involved in the operational HR activities in order to provide concise and appropriate advice as and when required. Further research is proposed to explore the new role of 'coach' or 'mentor' to determine the influence it has on the line managers doing the HR tasks, and whether it enhances the strategic position of the profession. Additionally, as discussed, Kryscynski et al (2018) show the increasing importance for HR professionals to obtain analytical skills, especially in an era when the business world is changing, further illustrating another role of an 'analyst' emerging. Therefore, the emerging role of 'mentor' and 'analyst' further portray the role of HR professionals as 'trendwatchers' as they will increasingly analyse the changes in the business climate and ensure that line managers, leadership team and the organisation as a whole is mentored in a manner where they are prepared to deal with the changes. The development of roles such as 'mentor' and 'analyst' along with Ulrich's role of strategic business partner, change agent, employee champion and administrative expert and the variation of these roles such as functional expert, HR leader, not only show the constant evolvement of the HR profession but also highlight the fact that a lot is possibly expected from HR professionals, consequently influencing their power and perception to various extents within organisations. Figure 3 shows an HR model in an ideal organisation, illustrating the necessity for all roles to connect for HR to successfully function in an organisation. Therefore, to be an HR leader, an HR professional needs to be an employee champion and a business partner, which can be enhanced by being a mentor and an analyst. Additionally, a successful HR leader will likely have the influence to execute a required change within the organisation. Further research is recommended to enhance the proposed model to thoroughly comprehend the value HR can bring to an organisation. 


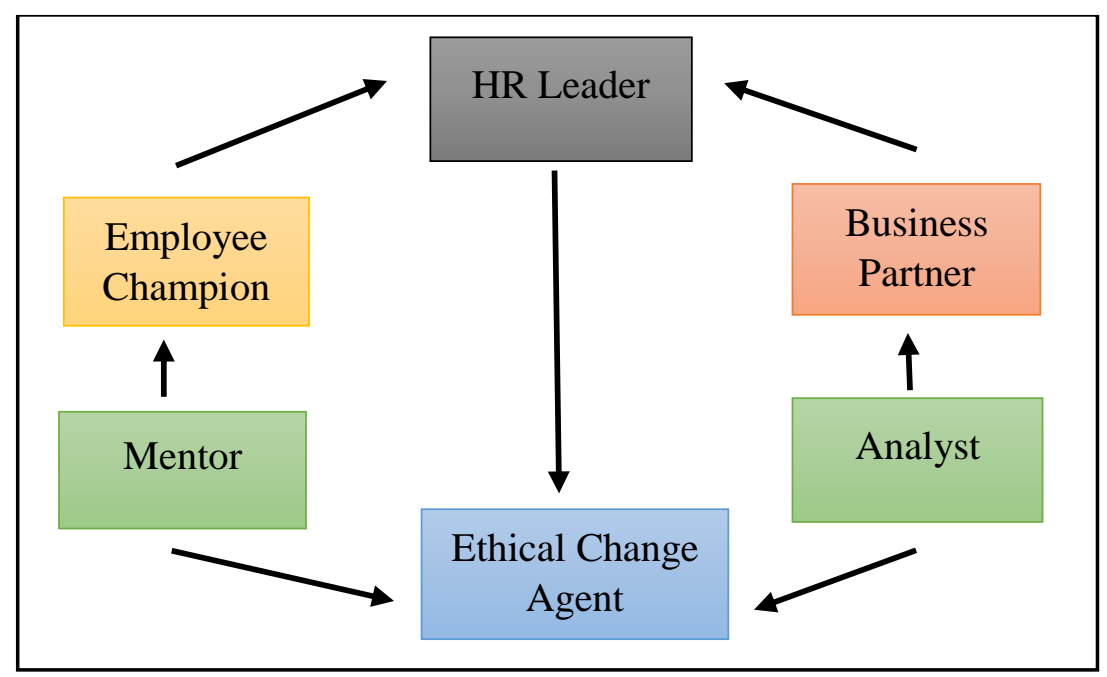

Figure 3 HR function in the ideal organisation

Finally, this study shows a clear recognition that the HR profession operates in a very complex environment as HR professionals are often required to tread in 'grey' situations which are not clear-cut and requires an in-depth investigation. Therefore, though this study does take into account the intricacy for HR professionals to deal with unethical situations, it also argues that if there is strong facilitation from the senior leaders, along with an appreciation for the HR function, it is possible for HR professionals to appropriately deal with an unethical issue. This further shows that HR professionals' emphasis on working for the business as ultimately it is about minimizing the risk that organisations may be vulnerable to, which includes the well-being of the employees. Therefore, though Caldwell (2003) show that there is an ongoing pressure on HR professionals to manage more than one role, this study argues that HR professionals put organisation as a priority, which doesn't just include the leadership team, but also the employees within the organisation, therefore portraying the employee champion role embedded within the strategic HR role. As already established in 5.3, HR professionals approached for this study were from organisations who strived to have an ethical culture, hence it cannot be assumed that every organisation understands the importance of HR. Considering the influence of organisational culture on HR professionals, further research on HR professionals in organisations who are lacking an understanding of the HR function should be explored to get an additional perspective on the profession and its future. 


\subsubsection{Practical Implications}

The changes in the business climate have made it increasingly important for organisations to ensure that their HR function is highly competent to deal with any arising complex situations. Additionally, with their gradual enhanced visibility at the senior leadership level, HR professionals themselves need to be more proactive, answer key questions with confidence, provide consistent and cohesive solutions and work collectively with other organisation members (Schiemann \& Ulrich, 2017) to be a key strategic player in the organisation. Considering the influence of role-conflict on HR professional's ability to decide between what is right for the organisation and their own morals (Linehan \& O’Brien, 2017), organisations and HR professionals need to have a strong working relationship in order to achieve outcomes that work in favour for both the organisation and employees. Given the complexity of the profession, HR professionals cannot solve it all, therefore increasing the need for a collaborative organisation culture to achieve strong partnerships and employee engagement (Schiemann \& Ulrich, 2017) that will then enhance organisation performance. This thesis provides practical suggestions for organisations and HR professionals to ensure that the HR profession can confidently deal with ethical situations and that they are ready for the future of work.

As no human being is the same, HR professionals are often expected to operate in shades of grey as different individuals have different interpretations of any one event. Given this complexity, an independent body within the organisation will be highly beneficial for HR professionals for when they need further guidance and direction on matters relating to ethical dilemmas and role-conflict. This independent body made up of different professionals such as a legal representative, an ethics officer and the CEO can provide HR professionals with a degree of independent advice in situations which may involve various organisation members. Organisations investing in an independent body for HR professionals will not only make decision-making more accurate, but it will also enhance organisation transparency and collectiveness which is highly needed in this turbulent business world. Furthermore, HR professionals should be strategic about who they build their relationships with. Therefore, engaging with organisation leaders who have a strong, positive influence within the organisation, will provide HR professionals with support from these leaders when they are feeling under attack or treading in extreme ambiguity.

With the future of work changing significantly, it will strongly be valuable for HR professionals to upskill and re-train to prepare for the changing demographics, the influence 
of technology and the increasing awareness of environment and sustainability issues. As discussed by Kryscynski et al (2017), advancing in analytical skills will provide HR professionals the opportunity to examine various trends and forecast predictions to ensure that the organisation policies and practices are up to date with the upcoming workforce changes. This is also likely to enhance the profession's strategic position within the organisation due to their "evidence-based decision-making" (Kryscynski et al, 2017, p. 715) as a result of increasing analytical ability. Furthermore, considering that there is an increasing necessity for a resilient partnership between a CE and the head of HR, recruiting certain leadership skills in an organisation will consequently have an impact on the HR function and the relationship between HR and the leadership team. This may also provide HR an opportunity to gain a seat at the management table while working closely with the business leaders to ensure they understand the influence of their decision-making on the peopleelement of the business. Considering this, organisations should ensure that they recruit a CEO that understands the significance and complexity of the HR function and the value it brings to the organisation. This is highly imperative due to the strong influence of organisation culture on the HR function; a culture which the CEO of the organisation is held responsible for.

\subsection{Conclusion}

It is increasingly evident that the future of work is shifting with the influences of globalisation, automation and generational cohorts which inevitably will have an influence on people's perception toward work and workplace behaviour. Considering that HR professionals are often known as the guardian of the organisation culture, the significance of the profession is strongly imperative as they will more than any other organisation function will be responsible for ensuring that the organisation and the employees are prepared for the future of work. This thesis concludes that with the increasing move to a strategic HR function, HR professionals are strongly working towards aligning the people-element of the business with the business objectives. Given that the HR function is embedded within the organisation culture, dealing with role-conflict in an ethical situation though challenging, will be practicable when there is an organisational culture with a strong perceived organisational support showing that senior leaders will provide guidance during uncertainty. Moreover, following a fair and thorough process where all situational factors are considered illustrates the relevance of moral development theory in further minimizing role-conflict within the profession. The move to a strategic position provides an opportunity for HR professionals to build a strong partnership with the business leaders and enables them to demonstrate their 
credibility by influencing business leaders. Therefore, with the growing strategic HR role, the recognition of the complexity of working in HR and the value they bring to enhance organisation performance, will possibly lead to a remuneration increase for the profession.

This study aligns with the existing HR literature in relation to the changing profession, with an emphasis of the influence of social learning theory in organisations. A strong example of culture is portrayed by Emmanuel Lulin from L'Oreal who said "companies' culture of ethics and integrity has become a substantial part of their assessment” (Markets Insider, 2018), hence making L'Oreal one of the most ethical companies in the world (Markets Insider, 2018). Furthermore, an organisation that promotes and thrives on unethical behaviour is likely to have an HR function that is unsuccessful at dealing with the unethical behaviour due to the top-down approach, hence affecting the quality of workplace relationships further highlighting the relevance of social exchange theory. Considering the impact of organisational culture on the HR function, the role theory also aligned with this study as HR professionals are often required to take on different roles' dependent upon the kind of stakeholder they are dealing with or the kind of situation they are required to resolve. Finally, the study adds to the literature by showing the growing emergence of the 'mentor' and 'analyst' roles, illustrating the shifting roles and the continuous evolvement within the HR profession. 


\section{References}

Avey, J. B., Wernsing, T. S., \& Palanski, M. E. (2012). Exploring the process of ethical leadership: The mediating role of employee voice and psychological ownership. Journal of Business Ethics, 107(1), 21-34.

Bai, Y., Lin, L., \& Liu, J. T. (2017). Leveraging the employee voice: a multi-level social learning perspective of ethical leadership. The International Journal of Human Resource Management, 1-33.

Bazley, D.M. (2018). Independent Review of Russell McVeagh. Retrieved from https://www.russellmcveagh.com/getmedia/cc682d64-46a1-40e3-987bcd82223bea24/Independent-Review-of-Russell-McVeagh-2018.pdf/

Beer, M. (1997). The transformation of the human resource function: Resolving the tension between a traditional administrative and a new strategic role. Human Resource Management: Published in Cooperation with the School of Business Administration, The University of Michigan and in alliance with the Society of Human Resources Management, 36(1), 49-56.

Biddle, B. J. (1986). Recent developments in role theory. Annual review of sociology, 12(1), 67-92.

Brian Picot Chair in Ethical Leadership. (2018). Ethical Leadership: Opportunities and Challenges for Aotearoa New Zealand. Retrieved from https://www.victoria.ac.nz/_data/assets/pdf_file/0003/1719471/FC0412_Brian-PicotChair_FINAL_web.pdf

Brown, M. E., \& Treviño, L. K. (2006). Ethical leadership: A review and future directions. The leadership quarterly, 17(6), 595-616.

Brown, M. E., Treviño, L. K., \& Harrison, D. A. (2005). Ethical leadership: A social learning perspective for construct development and testing. Organizational behavior and human decision processes, 97(2), 117-134.

Brown, M., Kulik, C. T., Cregan, C., \& Metz, I. (2017). Understanding the change-cynicism cycle: the role of HR. Human Resource Management, 56(1), 5-24.

Bush, J. T. (2018). Win-Win-Lose? Sustainable HRM and the promotion of unsustainable employee outcomes. Human Resource Management Review.

Bushnell, M. (2019, May 21). Business After \#MeToo: What's Changed and What Hasn't in the Business World. Retrieved June 2, 2019 from https://www.business.com/articles/me-toomovement-small-business/

Caldwell, R. (2003). The changing roles of personnel managers: old ambiguities, new uncertainties. Journal of Management Studies, 40(4), 983-1004.

Canedo, J. C., Graen, G., Grace, M., \& Johnson, R. D. (2017). Navigating the new workplace: Technology, millennials, and accelerating HR innovation. AIS Transactions on Human-Computer Interaction, 9(3), 243-260. 
Chiu, S. F., Yeh, S. P., \& Huang, T. C. (2015). Role stressors and employee deviance: the moderating effect of social support. Personnel Review, 44(2), 308-324.

Choi, Y., Kim, J. Y., \& Yoo, T. (2016). A study on the effect of learning organisation readiness on employees' quality commitment: the moderating effect of leader-member exchange. Total Quality Management \& Business Excellence, 27(3-4), 325-338.

Chung, F. (2019, March 13). Why millennials can't last 90 days at work. Retrieved June 11, 2019 from

https://www.nzherald.co.nz/business/news/article.cfm?c_id=3\&objectid=12212392

Cialdini, R. B. (2003). Crafting normative messages to protect the environment. Current directions in psychological science, 12(4), 105-109.

Cohen, L., \& Manion, L. (1994). Research methods in education. (4th ed.) London: Routledge.

Colmar Brunton (2018). Better Futures. Retrieved from

https://www.colmarbrunton.co.nz/wp-content/uploads/2019/02/Colmar-Brunton-BetterFutures-2019-MASTER-FINAL-REPORT.pdf

Cropanzano, R., \& Mitchell, M. S. (2005). Social exchange theory: An interdisciplinary review. Journal of management, 31(6), 874-900.

Davies, F. M., \& Gould-Williams, J. S. (2005). Using social exchange theory to predict the effects of HRM practice on employee outcomes. Public management review, 7(1), 1-24.

Deloitte. (2016). The Future of the Workforce. Critical drivers and challenges. Retrieved from https://www2.deloitte.com/global/en/pages/human-capital/articles/future-of-theworkforce.html

Foote, D., \& Robinson, I. (1999). The role of the human resources manager: strategist or conscience of the organisation?. Business Ethics: A European Review, 8(2), 88-98.

Fraedrich, J., Thorne, D. M., \& Ferrell, O. C. (1994). Assessing the application of cognitive moral development theory to business ethics. Journal of Business Ethics, 13(10), 829-838.

Francis, H., \& Keegan, A. (2006). The changing face of HRM: in search of balance. Human Resource management journal, 16(3), 231-249.

Guest, D. E., \& Woodrow, C. (2012). Exploring the boundaries of human resource managers' responsibilities. Journal of business ethics, 111(1), 109-119.

Guest, D., \& King, Z. (2004). Power, innovation and problem-solving: the personnel managers' three steps to heaven?. Journal of management studies, 41(3), 401-423.

Harrington, S., \& Rayner, C. (2010). Look before you leap or dive right in? The use of moral courage in response to workplace bullying. In Oxford handbook of positive psychology and work. 
Heres, L., \& Lasthuizen, K. (2012). What's the difference? Ethical leadership in public, hybrid and private sector organizations. Journal of Change Management, 12(4), 441-466.

IT Brief. (2019, May 08). Companies seeking digital transformation should focus on people. Retrieved June 08, 2019 from https://itbrief.co.nz/story/companies-seeking-digitaltransformation-should-focus-on-people

Jordan, J., Brown, M. E., Treviño, L. K., \& Finkelstein, S. (2013). Someone to look up to: Executive-follower ethical reasoning and perceptions of ethical leadership. Journal of management, 39(3), 660-683.

Kaeser, J. (2019, January 22). Globalization 4.0 - what to expect from leaders. Retrieved June 2, 2019 from https://www.weforum.org/agenda/2019/01/what-we-expect-from-leadersin-globalization-4-0/

Kaptein, M. (2011). From inaction to external whistleblowing: The influence of the ethical culture of organizations on employee responses to observed wrongdoing. Journal of Business Ethics, 98(3), 513-530.

Kaptein, M. (2011). Understanding unethical behavior by unraveling ethical culture. Human relations, 64(6), 843-869.

Khan, S. (2012). HR Devolution: A Review of the Literature. New Zealand Journal of Human Resources Management, 12(1).

Kohlberg, L. 1969. Stage and sequence: The cognitive-developmental approach to socialization. In D. A. Goslin (Ed.), Handbook of socialization and research: 347-480. Chicago: Rand McNally.

Kowalski, T. H., \& Loretto, W. (2017). Well-being and HRM in the changing workplace. The International Journal of Human Resource Management, 28(16), 2229-2255.

Kryscynski, D., Reeves, C., Stice-Lusvardi, R., Ulrich, M., \& Russell, G. (2018). Analytical abilities and the performance of HR professionals. Human Resource Management, 57(3), 715-738.

Kuipers, B. S., \& Giurge, L. M. (2017). Does alignment matter? The performance implications of HR roles connected to organizational strategy. The International Journal of Human Resource Management, 28(22), 3179-3201.

Lawton, A., Rayner, J., \& Lasthuizen, K. (2013). Ethics and Management in the Public Sector. Abingdon, Oxon: Routledge

Legge, K. (1978). Power, innovation, and problem-solving in personnel management. MacGraw-Hill Book Company.

Lemmergaard, J. (2009). From administrative expert to strategic partner. Employee Relations, 31(2), 182-196. 
Lengnick-Hall, M. L., Lengnick-Hall, C. A., Andrade, L. S., \& Drake, B. (2009). Strategic human resource management: The evolution of the field. Human resource management review, 19(2), 64-85.

Li, F., Chao, M. C. H., Chen, N. Y. F., \& Zhang, S. (2018). Moral judgment in a business setting: The role of managers' moral foundation, ideology, and level of moral development. Asia Pacific Journal of Management, 35(1), 121-143.

Linehan, C., \& O'Brien, E. (2017). From tell-tale signs to irreconcilable struggles: The value of emotion in exploring the ethical dilemmas of human resource professionals. Journal of Business Ethics, 141(4), 763-777.

Lo, K., Macky, K., \& Pio, E. (2015). The HR competency requirements for strategic and functional HR practitioners. The International Journal of Human Resource Management, 26(18), 2308-2328.

Manz, C. C., \& Sims Jr, H. P. (1981). Vicarious learning: The influence of modeling on organizational behavior. Academy of Management Review, 6(1), 105-113.

Marchington, M. (2015). Human resource management (HRM): Too busy looking up to see where it is going longer term?. Human Resource Management Review, 25(2), 176-187.

Markets Insider. (2018, February 13). L'Oreal named as one of the World's Most Ethical Companies by the Ethisphere Institute for the 9th time. Retrieved June 15, 2019 from https://markets.businessinsider.com/news/stocks/1-oreal-named-as-one-of-the-world-s-mostethical-companies-by-the-ethisphere-institute-for-the-9th-time-1015605347

McCribb, J. (2018, August 17). Jo Cribb: Russell McVeagh report a wake-up call for many. Retrieved June 1, 2019 from https://www.nzherald.co.nz/nz/news/article.cfm?c_id=1\&objectid=12108181

Mitchell, S. (2019, May 5). Ethics good for the triple bottom line, says L'Oreal executive. Retrieved June 2, 2019 from https://www.afr.com/business/retail/ethics-good-for-the-triplebottom-line-says-1-oreal-executive-20190429-p51idb

Moon, K., \& Blackman, D. (2014). A guide to understanding social science research for natural scientists. Conservation Biology, 28(5), 1167-1177.

Moon, K., Brewer, T., Januchowski-Hartley, S., Adams, V., \& Blackman, D. (2016). A guideline to improve qualitative social science publishing in ecology and conservation journals. Ecology and Society, 21(3).

Mullins, F. (2018). HR on board! The implications of human resource expertise on boards of directors for diversity management. Human Resource Management, 57(5), 1127-1143.

Neubert, M. J., Carlson, D. S., Kacmar, K. M., Roberts, J. A., \& Chonko, L. B. (2009). The virtuous influence of ethical leadership behavior: Evidence from the field. Journal of Business Ethics, 90(2), 157-170. 
Newell, C. (2017, August 9). Why every CEO should give HR a seat at the table. Retrieved June 2, 2019 from

https://www.forbes.com/sites/forbeshumanresourcescouncil/2017/08/09/why-every-ceoshould-give-hr-a-seat-at-the-table/\#2b9dc2835bd0

Newsroom. (2018, October 11). What really happened at the Russell McVeagh's Bar. Retrieved June 2, 2019 from https://www.newsroom.co.nz/2018/10/10/273174/russellmcveagh-partner-steps-down-over-bullying-claims\#

O'Leary, Z. (2017). The essential guide to doing your research project. 3rd Ed., London, UK: Sage

O'Brien, E., \& Linehan, C. (2014). A balancing act: Emotional challenges in the HR role. Journal of Management Studies, 51(8), 1257-1285.

Parkes, C., \& Davis, A. J. (2013). Ethics and social responsibility-do HR professionals have the 'courage to challenge' or are they set to be permanent 'bystanders?'. The International Journal of Human Resource Management, 24(12), 2411-2434.

Parzefall, M. R., \& Salin, D. M. (2010). Perceptions of and reactions to workplace bullying: A social exchange perspective. Human Relations, 63(6), 761-780.

Riivari, E., \& Lämsä, A. M. (2014). Does it pay to be ethical? Examining the relationship between organisations' ethical culture and innovativeness. Journal of Business Ethics, 124(1), 1-17.

Rizzo, J. R., House, R. J., \& Lirtzman, S. I. (1970). Role conflict and ambiguity in complex organizations. Administrative science quarterly, 150-163.

Schiemann, W. A., \& Ulrich, D. (2017). Rise of HR-New mandates for IO. Industrial and Organizational Psychology, 10(1), 3-25.

Schindler, J. (2019, January 07). Leading With Ethics. Retrieved June 11, 2019 from https://www.forbes.com/sites/forbescoachescouncil/2019/01/07/leading-withethics/\#a015e77568ad

Schwandt, T. A. (1994). Constructivist, interpretivist approaches to human inquiry. Handbook of qualitative research, 1, 118-137.

Sheehan, C., De Cieri, H., Greenwood, M., \& Van Buren III, H. J. (2014). HR professional role tensions: Perceptions and responses of the top management team. Human Resource Management, 53(1), 115-130.

Shivers-Blackwell, S. L. (2004). Using role theory to examine determinants of transformational and transactional leader behavior. Journal of Leadership \& Organizational Studies, 10(3), 41-50.

Stone, D. L., \& Deadrick, D. L. (2015). Challenges and opportunities affecting the future of human resource management. Human Resource Management Review, 25(2), 139-145. 
Storey, J. (1992). Developments in the Management of Human Resources. Oxford: Blackwell Publishing.

Sumpter, D. M., Gibson, C. B., \& Porath, C. (2017). Act expediently, with autonomy: Vicarious learning, empowered behaviors, and performance. Journal of Business and Psychology, 32(2), 131-145.

Taulli, T. (2019, May 4). How To Reskill Your Workforce For AI (Artificial Intelligence). Retrieved June 2, 2019 from https://www.forbes.com/sites/tomtaulli/2019/05/04/how-toreskill-your-workforce-for-ai-artificial-intelligence/\#2aa79a523eb9

Tett, G. (2019, May 24). More chief executives are paying for their ethical mis-steps. Retrieved June 2, 2019 from https://www.ft.com/content/490c12e4-7cab-11e9-81d2$\underline{\mathrm{f7} 85092 \mathrm{ab} 560}$

Trevino, L. K. (1986). Ethical decision making in organizations: A person-situation interactionist model. Academy of management Review, 11(3), 601-617.

Trevino, L. K., Hartman, L. P., \& Brown, M. (2000). Moral person and moral manager: How executives develop a reputation for ethical leadership. California management review, 42(4), 128-142.

Ulrich, D. (1997). Human Resource Champions. Boston: Harvard University Press

Ulrich, D. (1998). A new mandate for human resources. Harvard business review, 76, 124135 .

Ulrich, D., \& Brockbank, W. 2005. The HR value proposition. Boston: Harvard Business School Press

Ulrich, D., \& Dulebohn, J. H. (2015). Are we there yet? What's next for HR?. Human Resource Management Review, 25(2), 188-204.

Van der Wal, Z. (2017). The $21^{\text {st }}$ Century Public Manager. London, UK: Palgrave

Vance, A. (2019, May 21). Parliament is a toxic workplace with a systematic bullying problem - Francis Review. Retrieved June 2, 2019 from

https://www.stuff.co.nz/national/politics/112865411/parliament-a-toxic-workplace-withsystematic-bullying-problem--francis-review

Wayne, S. J., Shore, L. M., \& Liden, R. C. (1997). Perceived organizational support and leader-member exchange: A social exchange perspective. Academy of Management journal, $40(1), 82-111$.

Weaver, G. R., \& Trevino, L. K. (2001). The role of human resources in ethics/compliance management: A fairness perspective. Human Resource Management Review, 11(1-2), 113134.

Welch, C. L., \& Welch, D. E. (2012). What do HR managers really do?. Management International Review, 52(4), 597-617. 
Whitener, E. M., Brodt, S. E., Korsgaard, M. A., \& Werner, J. M. (1998). Managers as initiators of trust: An exchange relationship framework for understanding managerial trustworthy behavior. Academy of management review, 23(3), 513-530.

Yin, Z. R. (2011). Qualitative Research from Start to Finish. New York, USA: The Guilford Press. 


\section{Appendices}

\subsection{Appendix 1 - Interview Protocol}

\begin{tabular}{|c|c|}
\hline \multicolumn{2}{|l|}{ Introduction } \\
\hline \multicolumn{2}{|c|}{$\begin{array}{l}\text { - Intro of myself } \\
\text { - Intro of the research topic: How do HR professionals cope with role-conflicts when } \\
\text { they experience ethical situations in organisations? How do these coping strategies } \\
\text { during an ethical situation impact the profession? } \\
\text { - Overview of the interview, make the participant aware that I will taking notes } \\
\text { - Ensure they know and provide consent for the interview to be recorded } \\
\text { - Interviewee will receive the transcript }\end{array}$} \\
\hline \multicolumn{2}{|c|}{ Can you tell me a bit about yourself and your background? } \\
\hline $\begin{array}{l}\text { - } \text { Career history } \\
\text { - } \text { Reason for choosing a career in HR } \\
\text { A bit about the current } \\
\text { organisation: size, structure, culture } \\
\text { - Current role: title, job description, } \\
\text { over-all responsibilities }\end{array}$ & $\begin{array}{l}\text { - What do you enjoy most about your } \\
\text { role? } \\
\text { - What are some of the main challenges } \\
\text { in your role? }\end{array}$ \\
\hline \multicolumn{2}{|c|}{ Introduce role-conflict - Do you experience role-conflict in your current role? } \\
\hline $\begin{array}{l}\text { According to literature by management } \\
\text { scholars, HR professionals have four main } \\
\text { roles that they must comply with; } \\
\text { Administrative Expert, Change Agent, } \\
\text { Strategic Business Partner and Employee } \\
\text { Advocate. The literature highlights that } \\
\text { each of these roles are unique on their own } \\
\text { and different from each other, hence } \\
\text { potentially creating a bit of a conflict for } \\
\text { the HR professional. } \\
\text { - Range of roles } \\
\text { - Role conflict/role tension } \\
\text { - Resolution procedure }\end{array}$ & $\begin{array}{l}\text { - What sort of roles do you undertake } \\
\text { in your current role? } \\
\text { - } \\
\text { Due to these various roles that you are } \\
\text { required to undertake, do you } \\
\text { experience role conflict or role } \\
\text { tension? } \\
\text { - What were some of the factors that } \\
\text { created the tension? } \\
\text { - In your opinion, what factors are } \\
\text { required to reduce the level of HRM } \\
\text { role tension? } \\
\text { Considering the role-conflict you } \\
\text { experience, how do you/would you } \\
\text { usually go about resolving issues } \\
\text { regarding employees, management or } \\
\text { the organisation as a whole? } \\
\text { Could you elaborate further or } \\
\text { provide some specific examples? }\end{array}$ \\
\hline
\end{tabular}




\begin{tabular}{|c|c|}
\hline \multicolumn{2}{|c|}{$\begin{array}{l}\text { Have you dealt with a difficult or sensitive situation at work? Eg: performance } \\
\text { management, behavioural misconduct, interpersonal or organisational deviance }\end{array}$} \\
\hline $\begin{array}{l}\text { - } \text { Unethical situation } \\
\text { - } \text { Features of unethical situation } \\
\text { - } \text { Reactions, emotions } \\
\text { - } \text { Results and actions } \\
\text { - Morals and beliefs } \\
\text { - } \quad \text { Relationship between role-conflict } \\
\text { and unethical situations } \\
\text { - Influence on the HR profession }\end{array}$ & 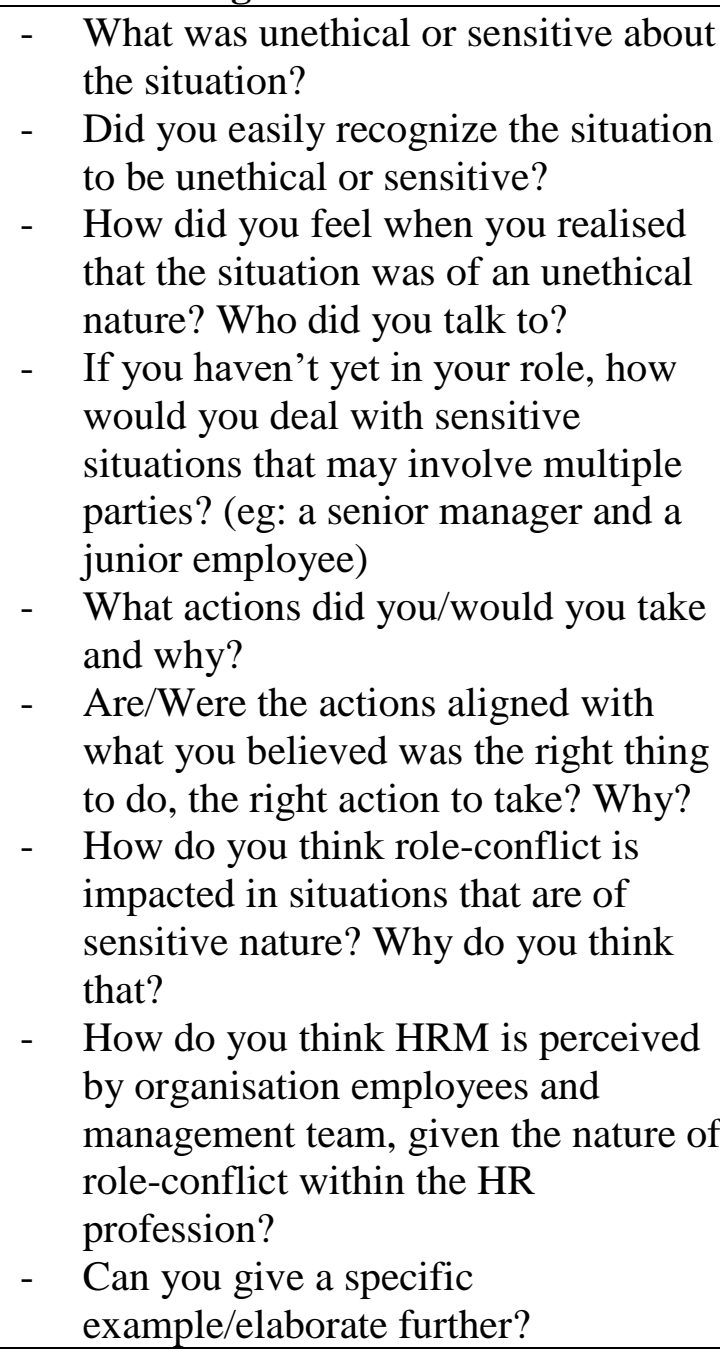 \\
\hline \multicolumn{2}{|l|}{ Recommendations and Best Practices } \\
\hline $\begin{array}{l}\text { - Emphasis on 'employee advocate' } \\
\text { and 'strategic business partner' } \\
\text { - Resolving role-conflict } \\
\text { - Opinion on the profession }\end{array}$ & $\begin{array}{l}\text { - What would you say is your best } \\
\text { practice when dealing with the role- } \\
\text { conflict between the 'employee } \\
\text { advocate' and 'strategic business } \\
\text { partner' role? } \\
\text { - Can you explain further? } \\
\text { - Would these best practices also be } \\
\text { suitable for role-conflicts in ethical } \\
\text { situations? Would you deal with it } \\
\text { differently? Why? } \\
\text { - What do you think HR professionals } \\
\text { in organisations should be responsible } \\
\text { for? Why? } \\
\text { - How important is the role of HR } \\
\text { professionals? }\end{array}$ \\
\hline \multicolumn{2}{|c|}{$\begin{array}{l}\text { End of the interview. Is there anything else you would like to add that we haven't } \\
\text { covered already? }\end{array}$} \\
\hline
\end{tabular}




\subsection{Appendix 2 - Letter to participants}

Date

Name

Title

Company

Dear XX,

HR professionals in organisations play an integral role where they are responsible for employee well-being, managing mostly confidential information, operating as strategic partners for leadership and governance and acting as change agents during restructuring processes in the organisation. These different roles might be difficult to combine, even more so in a time where traditional business protocols and practices sometimes seem outdated. For example, millennials who have entered the workforce have challenged traditional ways of work with their emphasis on achieving meaningful work experiences. Hence, it has increasingly become imperative for organisations to attain an ethical and safe organisational culture.

As Victoria University of Wellington's Masters' student, I will conduct a study as part of my thesis "HR \& Ethics. A relationship that is work in progress" with the guidance of my supervisor Prof Karin Lasthuizen. I will study how HR professionals from a range of organisations cope with role conflict and reconcile different organisational demands, how these different demands influence the relationship between HR and ethics in the workplace, and how it ultimately shapes today's HR profession. I am particularly interested to find out what the best practices are when HR professionals try to navigate in ethical situations or dilemmas and when there are conflicting roles and/or demands.

Considering your position and expertise, I am interested to hear your experiences and thoughts with regards to working in a HR position within \{organisation name\}. I hope that you are willing to help me with my research; I will provide you with a topic list and a more detailed participant information sheet before the actual interview. Your contribution will very much be valued.

The interview will be approximately for one hour and can take place at your work, or if you prefer, at the Brian Picot Chair in Ethical Leadership within Victoria Business School.

My research has been approved by the Victoria University Human Ethics Committee with approval number (0000026924). The information you provide me will be handled in strictest of confidence and in the final report, your identity and the organisation will not be known.

Given the nature of your role, I am sure you are likely to be very busy and hence it will take time to book an interview. I am happy to work around your schedule and if required, I can 
liaise with your assistant to book in a time. I would ideally like to conduct interviews before May 2019. If you have any additional queries, please do not hesitate to contact me or my supervisor.

I look forward to hearing your thoughts.

Sincerely,

Saloni Pandey

\section{Contact details -}

Saloni Pandey

(Email \& Phone)

\section{Contact Supervisor -}

Professor Karin Lasthuizen

(Email \& Phone)

Should you have any ethics queries, you are welcome to contact HEC Convener -

Dr Judith Loveridge

(Email \& Phone) 


\subsection{Appendix 3 - Coding process}

Once the interviews were transcribed, the transcripts were colour coded to determine specific themes and patters. There were four pre-determined themes that derived out of the literature and research question; role-conflict, ethical situation, best practices and HR role. Considering the pre-determined themes, codes such as 'challenges', 'personal values', 'organisation structures' and 'future HR' were allocated to the themes, by establishing where they fit best. For example, when participants expressed views on how HR could be prepared for future, they were coded as 'future' or 'HR characteristics' and categorised under the theme of HR role. In this way, the interview transcripts were restructured with the most relevant findings organised per theme and code (Yin, 2011).

\begin{tabular}{|c|l|}
\hline Themes & \multicolumn{1}{|c|}{ Codes } \\
\hline \multirow{3}{*}{ Role-conflict } & A - Background \\
& B - Role \\
& C - Challenges \\
\hline \multirow{3}{*}{ Ethical situations } & $\begin{array}{l}\text { E - Organisation structure/expectation } \\
\text { K - Relationships/Behaviours/Actions }\end{array}$ \\
& II - Personal view/values/morals \\
Best Practices & G - Recommendations \\
\hline \multirow{2}{*}{ Role of HR } & $\begin{array}{l}\text { F - People's view on HR } \\
\text { H - Future } \\
\end{array}$ \\
\hline
\end{tabular}

